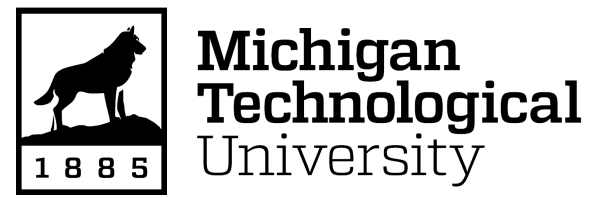

Michigan Technological University Digital Commons @ Michigan Tech

Dissertations, Master's Theses and Master's Reports

2017

\title{
CONSENSUS BASED DISTRIBUTED CONTROL IN MICRO-GRID CLUSTERS
}

Syed Ahmed Fuad

Michigan Technological University, sfuad@mtu.edu

Copyright 2017 Syed Ahmed Fuad

\section{Recommended Citation}

Fuad, Syed Ahmed, "CONSENSUS BASED DISTRIBUTED CONTROL IN MICRO-GRID CLUSTERS", Open Access Master's Thesis, Michigan Technological University, 2017.

https://doi.org/10.37099/mtu.dc.etdr/334

Follow this and additional works at: https://digitalcommons.mtu.edu/etdr

Part of the Power and Energy Commons 
CONSENSUS BASED DISTRIBUTED CONTROL IN MICRO-GRID CLUSTERS

By

Syed Ahmed Fuad

\section{A THESIS}

Submitted in partial fulfillment of the requirements for the degree of MASTER OF SCIENCE

In Electrical Engineering

\section{MICHIGAN TECHNOLOGICAL UNIVERSITY}

2017

(C) 2017 Syed Ahmed Fuad 
This thesis has been approved in partial fulfillment of the requirements for the Degree of MASTER OF SCIENCE in Electrical Engineering.

Department of Electrical and Computer Engineering

\author{
Thesis Co-Advisor: $\quad$ Sumit Paudyal \\ Thesis Co-Advisor: $\quad$ Mohsen Azizi
}

Committee Member: $\quad$ Wayne W. Weaver

Department Chair: Daniel R. Fuhrmann 


\section{Dedication}

To my parents, my teachers and my dearest friends. 


\section{Table of Contents}

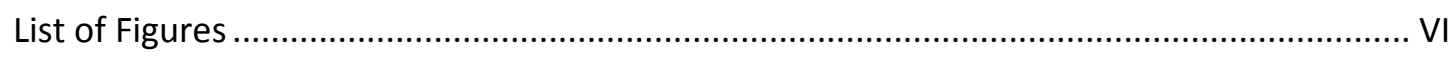

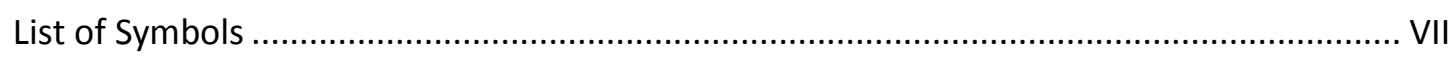

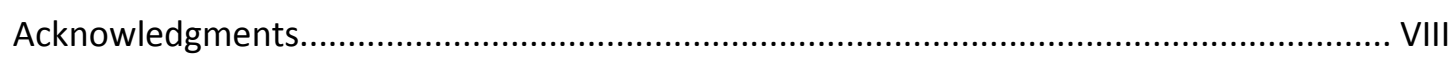

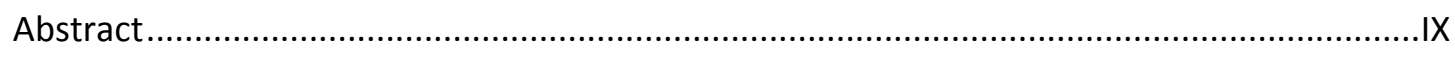

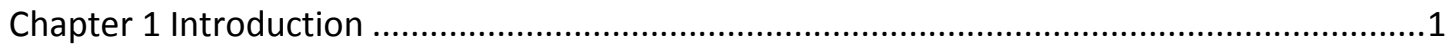

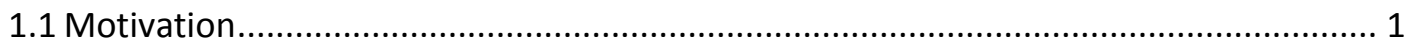

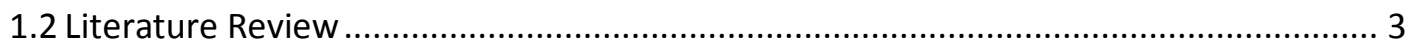

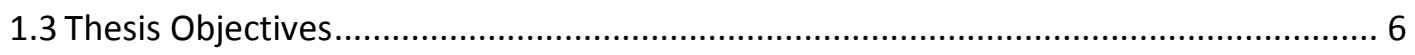

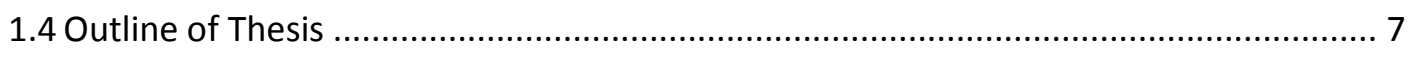

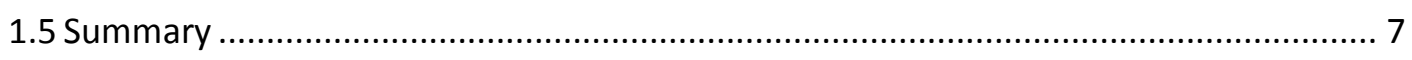

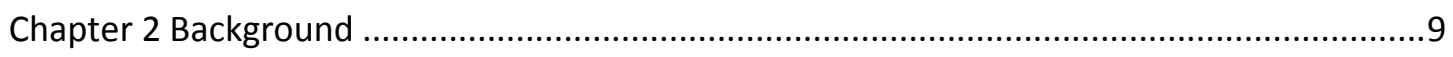

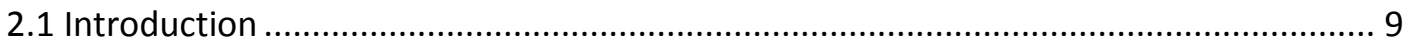

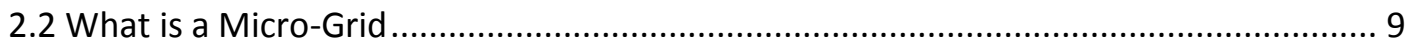

2.3 Relationship Between Reactive Power and Voltage Stabilization in Micro-Grids ........ 12

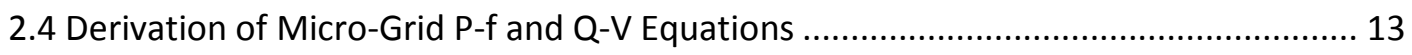

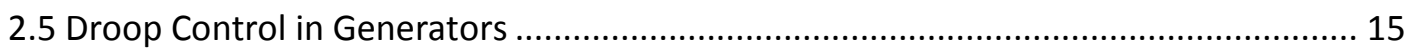

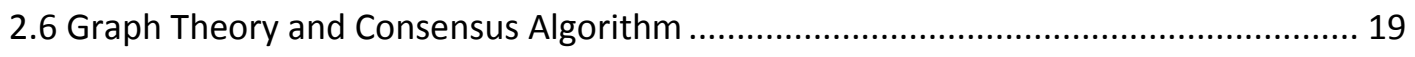

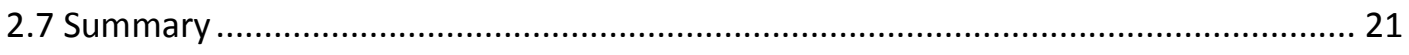

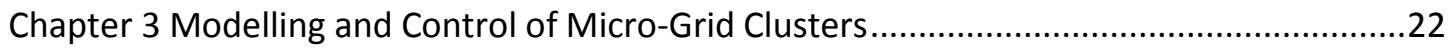

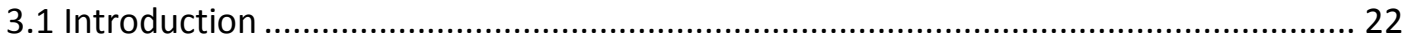

3.2 Describing Micro-Grids in Commercial Power Flow Software.................................... 22

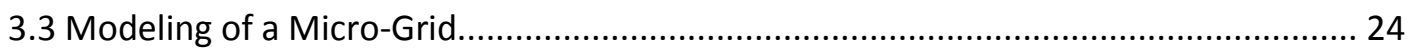

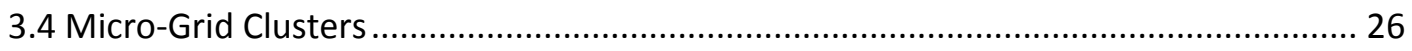

3.5 Proposed Consensus Based Droop Controller for Voltage Stabilization ..................... 27

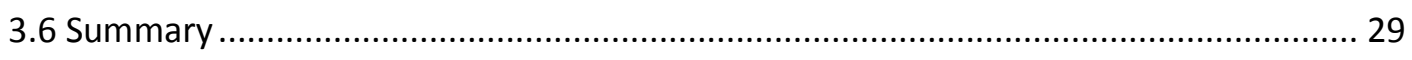

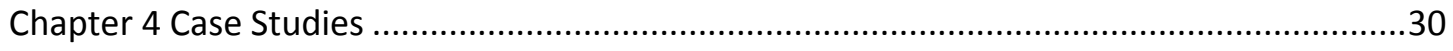

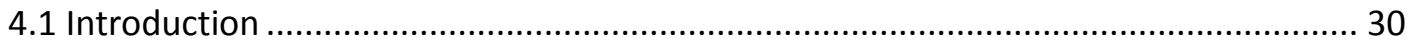

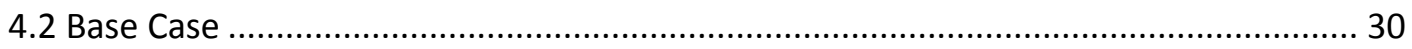


4.3 Consensus Control with Different Communication Topologies.................................. 31

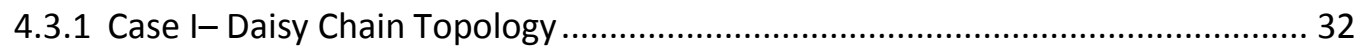

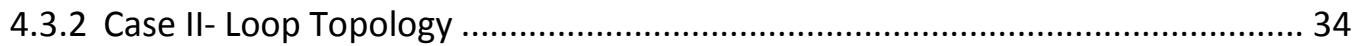

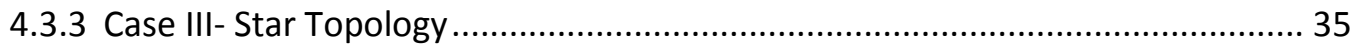

4.4 Performance of Consensus Control with Communication Time Delays ....................... 37

4.4.1 Case I- 0.5 Second Communication Delay ....................................................... 37

4.4.2 Case II- 1 Second Communication Delay ............................................................ 38

4.4.3 Case III- 3 Seconds Communication Delay .......................................................... 39

4.5 Effect of Generator Tripping on the Performance of Consensus Control .................... 40

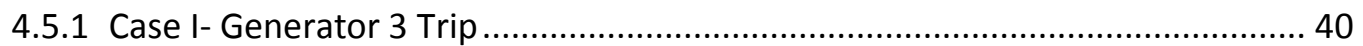

4.5.2 Case II- Generator 3 Trip: Corrective Control ................................................... 41

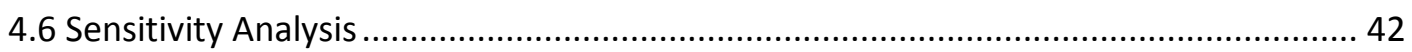

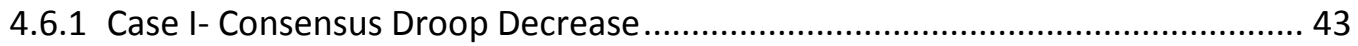

4.6.2 Case II- Consensus Droop Increase ................................................................. 44

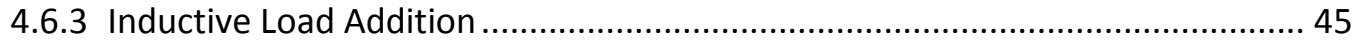

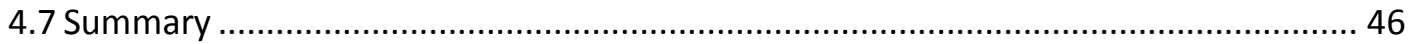

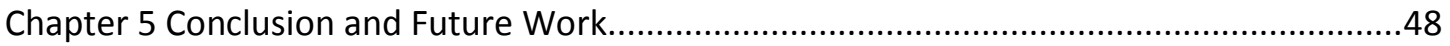

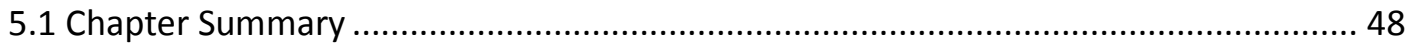

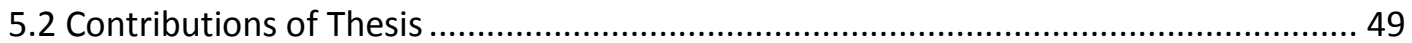

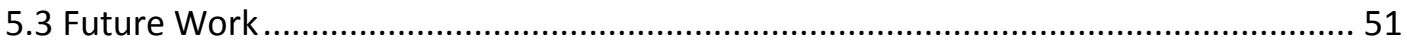

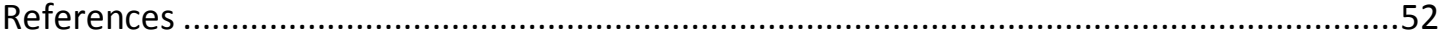

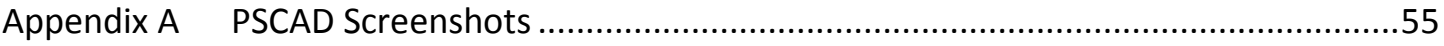




\section{List of Figures}

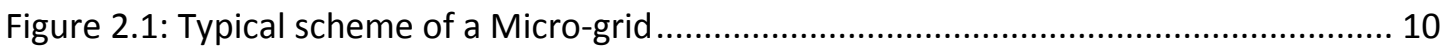

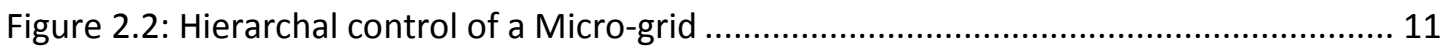

Figure 2.3: A simple current carrying electrical transmission line....................................... 13

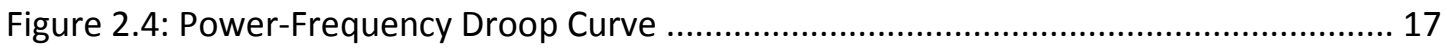

Figure 2.5: Reactive Power- Voltage Droop Curve ............................................................ 18

Figure 3.1: Model of a Micro-Grid under consensus control.............................................. 25

Figure 3.2: Single line diagram for the Micro-grid clusters under consensus control ............ 27

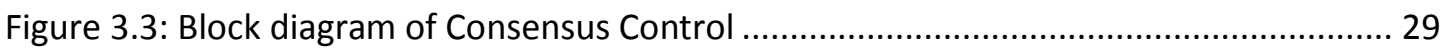

Figure 4.1: Comparison of micro-grid voltages with no consensus control ........................... 31

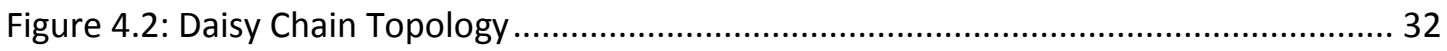

Figure 4.3: Comparison of Bus voltages with Daisy Chain communication topology ............. 32

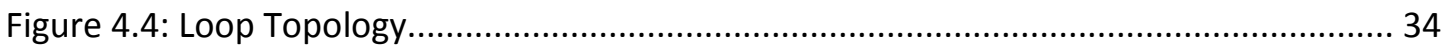

Figure 4.5: Comparison of Bus Voltages when all generators connected in a Loop................ 34

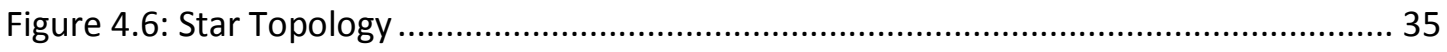

Figure 4.7: Bus Voltages when all generators connected in star topology ............................ 36

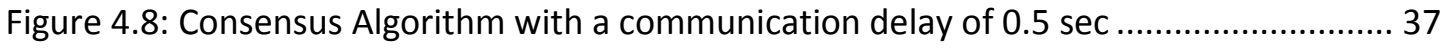

Figure 4.9: Consensus Algorithm with a communication delay of $1 \mathrm{sec}$.............................. 38

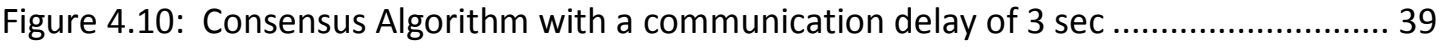

Figure 4.11: Effect of tripping of a generator in consensus control ..................................... 40

Figure 4.12: Corrective control due to the tripping of a generator ..................................... 42

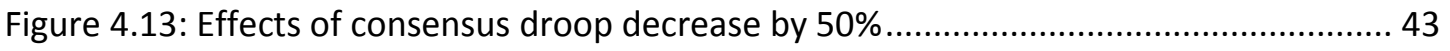

Figure 4.14: Effects of consensus droop increase by 50\% ................................................. 44

Figure 4.15: Voltage drop on each bus when 20 MVAR is added to each Micro-grid............ 45

Figure A.1: Complete Micro-Grid Cluster Model in PSCAD ................................................. 55

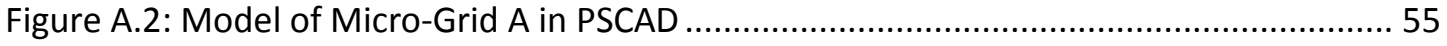

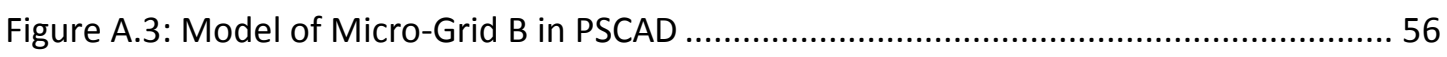

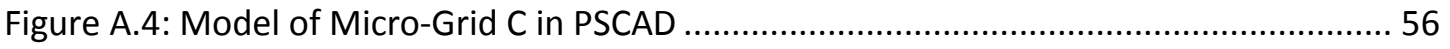

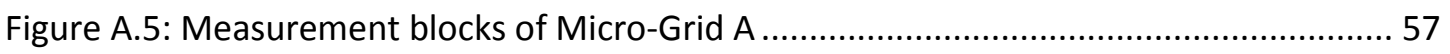

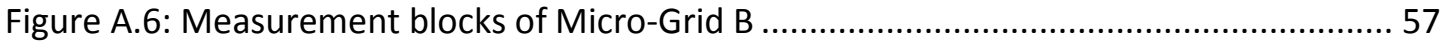

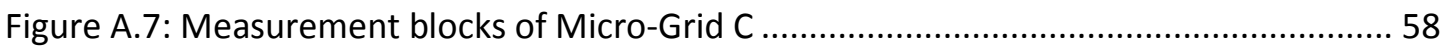

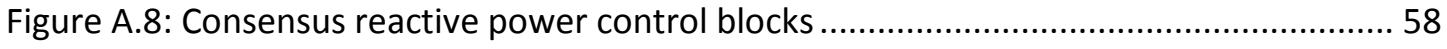

Figure A.9: Active power and Islanding mode control blocks............................................. 59 


\section{List of Symbols}

$D_{v} \quad$ Generator voltage droop capability

$D_{s} \quad$ Generator speed droop capability

$P_{0} \quad$ Nominal active power

$S_{p} \quad$ Slope of the P-f curve

$\mathrm{S}_{\mathrm{nl}} \quad$ No load speed of the generator

$\mathrm{S}_{\mathrm{fl}} \quad$ Full load speed of the generator

$f_{n l} \quad$ No load frequency of the generator

$f_{\text {sys, }} \quad$ Frequency of the system

$f_{0} \quad$ Nominal frequency

$Q \quad$ Reactive power output of the generator

$Q_{0} \quad$ Nominal reactive power

$\mathrm{S}_{\mathrm{q}} \quad$ Slope of the Q-V curve

$\mathrm{V}_{\mathrm{nl}} \quad$ No load voltage of the generator

$\mathrm{V}_{\mathrm{fl}} \quad$ Full load voltage of the generator

$V_{\text {sys }} \quad$ Nominal voltage of the system

$P \quad$ Active power of the generator

$f \quad$ Frequency of the generator

$I^{*} \quad$ Conjugate of phasor current

$S \quad$ Total apparent power

$Y \quad$ Admittance

$\delta \quad$ Power angle

$\dot{x}_{i} \quad$ System of element $x_{i}$

$u_{i} \quad$ Output of a system

$V_{G} \quad$ Set of finite and non-empty elements called nodes or vertices

$E_{G} \quad$ Set of unordered two elements of vertices, VG, called edges

G Graph

A $_{G} \quad$ Adjacency matrix

$\mathbf{D}_{\mathrm{G}} \quad$ Degree matrix

$a_{i j} \quad$ Entry of adjacency matrix

$\mathrm{L}_{\mathrm{G}} \quad$ Laplacian matrix

$l_{i j} \quad$ Entry of Laplacian matrix

$\in \quad$ Time step 


\section{Acknowledgments}

I would like to express my sincerest gratitude to my advisors, Dr. Sumit Paudyal and Dr. Mohsen Azizi, for their guidance and support throughout my research work. I would also like to thank my committee member, Dr. Wayne Weaver. This thesis would not have been possible without the support of the Fulbright Scholar Program and my Fulbright Program Officer, Ms. Stephanie Sasz, at the Institute of International Education. Lastly, I would like to thank my parents and my friends for their continuous support and encouragement. 


\section{Abstract}

With the increasing trend of utilizing renewable energy generators such as photovoltaic (PV) cells and wind turbines, power systems are transforming from a centralized power grid structure to a cluster of smart micro-grids with more autonomous power sharing capabilities. Even though the decentralized control of power systems is a reliable and cost effective solution, due to the inherent heterogeneous nature of micro-grids, optimal and efficient power sharing among distributed generators ( $D G^{\prime} s$ ) is a major issue which calls for advanced control techniques for voltage stabilization of the entire micro-grid cluster. The proposed consensus based algorithm in this thesis is a solution to overcome these control challenges, which only requires each DG to exchange information with its directly connected neighboring DG's, in order to maintain the power balance and voltage stability of the entire micro-grid cluster. The proposed method in this thesis is simulated in PSCAD and its effectiveness is demonstrated using several realistic and practical cases including micro-grid topology changes, communication delays, and load changes. 


\section{Chapter 1 Introduction}

\subsection{Motivation}

Electrical power has slowly evolved to become one of the basic necessities of life. From everyday household, common appliances to large complicated industrial plants, all require electrical power to run them. It is therefore highly important to safely generate and control electrical power so that it reaches its designated end user in a stable and reliable manner. The increase in awareness of global warming due to the carbon emissions of fossil fuel burning as well as the reduction in the amount of available fossil fuel reserves, has put the focus of many researchers from all over the world to find cleaner, and more efficient and reliable sources of energy. As the world gradually shifts its focus to greener and more environment friendly energy, electrical power generators have also transformed themselves from using fossil fuels to abundant clean energy sources. These clean energy sources, termed as 'Renewable Energy Sources', are based on several different physical and environmental phenomena such as wind, solar, tidal, wave, geothermal, biofuel, etc. Most of the renewable sources can efficiently harvest energy in small amounts as compared to conventional fossil fuel based energy sources. However, renewable energy sources can be installed in large numbers in a much wider area since they are more flexible in comparison to conventional power plants in terms of suitable locations for installation and cheaper in the initial investment costs [1].

Renewable energy sources might be cleaner, available for free and have no associated fuel transportation costs. However, due to their dependency on the weather, season and the time of the day, they are not reliable [2]. That added to the 
fact that electrical systems are becoming more efficient and smarter every day, makes the reliable control of these renewable energy sources more difficult and challenging. When these renewable energy sources are connected together or with an existing power distribution network, these energy sources are called 'Distributed Generators' (DGs) or 'Distributed Energy Resources' (DERs) [3]. These new distributed generators are much smaller in size and ratings, yet are very efficient in their performances and combine together to form small electrical networks called 'Micro-Grids' (MGs). The issue at hand is the control, integration and electrical stability of these micro-grids that is still under study.

With the addition of distributed generators in the power distribution system, a centralized control of the real and reactive power dispatch is neither cost effective nor practical. Therefore, the common practice adopted for the decentralized control of power sharing among the distributed generators include the traditional simple droop control for both real power-frequency and reactive power-voltage among the distributed generators which would work the same way as the control of synchronous generators. This usually works fine for the real power sharing and frequency control but the performance of grid voltage stabilization and reactive power sharing deteriorates which results in an unstable grid [4-6].

Some control strategies for reactive power have been proposed earlier that are basically based upon Lyapunov functions which result in the damping of the system voltages using just the local measured parameters. But the stability analysis has been done for small signal deviations and the complete droop control in an autonomous micro-grid has not been properly analyzed and investigated. It has been observed that the reactive power sharing is not efficient under large load variations and hence further study is required to deal with this issue [4].

Considering the above problem, the consensus based multi-agent control theory seems to be very promising and worth investigating. The goal of the consensus 
control involves a similar problem of autonomous control of many similar units or 'agents' working together in order to reach a certain 'consensus' to achieve the desired system performance. Interactions with only the directly connected neighboring generators is required for the consensus based algorithm to work, which makes the system very robust and reliable to large load variations [7].

\subsection{Literature Review}

The investment in micro-grids with several distributed generators would require substantial restructuring of the conventional power infrastructure. Most importantly, control and scheduling systems need to be changed from a centralized structure to a more distributed and autonomous system, as the former faces serious challenges of privacy, vulnerability of the failure of the central controller, and the vulnerability of the communication network due to the exponential increase of distributed generators in the system [8]. The distributed generators are of varied nature and can be physically placed in wide ranging locations and hence a much robust and reliable method of distributed control and management algorithm is required to ensure the overall security, stability and scalability of the micro-grid [6]. The consensus based algorithm is an excellent solution for the above challenges as it can easily bring all the generators in the micro-grid to a global agreement of a particular parameter of the generators using only neighbor to neighbor communication among them [8].

The Consensus algorithm has been studied for a very long time and was first introduced by DeGroot in 1960s in the fields of statistics and management science. Since then they have played a crucial part in computer science and the success related to distributed computing [7]. The same algorithm has been researched and is 
being used in different fields including robotics [9], autonomous vehicles [10], wireless sensors [11] and unmanned aerial vehicles [12].

In recent years, the consensus based algorithm has also been studied for electrical smart grids. The areas researched in smart grids include real power sharing between distributed generation inverters [13], control of virtual synchronous generators [14], load shedding [15] and economic load dispatch [16]. However, the grid voltage stabilization and the control of reactive power sharing has not been properly addressed yet [4].

The conventional droop based algorithms are still considered by many to be the most effective in terms of reactive grid voltage stability and reactive power sharing. This is due to the fact that the droop controllers are simpler in nature, require no external communication, are basically decentralized, and their performance is similar to conventional synchronous generators [17-19]. To further improve the droop controller and make its performance even closer to that of a conventional synchronous generator, virtual inertial load has been proposed to be incorporated with the droop inverters, resulting in a system that also satisfies the swing equation and would behave like conventional generators in transient characteristics as well. These inverters are then called 'Virtual Synchronous Generators' [20].

The conventional droop based control algorithms might be appealing but has its significant drawbacks as well. Apart from the load dependent frequencies and voltage amplitude, a micro-grid has distribution lines with a low $X / R$ ratio, which are mainly resistive, but the outputs of the distributed generators are predominantly inductive $[21,22]$. This impedance mismatch does not affect the real power sharing much but it results in the inaccurate reactive power sharing, giving rise to the voltage and grid stability issues [23]. The impedance output of the distributed generators can be virtually changed as well. But for proportional, stable and reliable power sharing, some degree of external communication is required since the load 
changes have a significant effect on the $X / R$ ratio of the micro-grid as well. Therefore, the consensus based algorithm is a control technique which requires minimal communication infrastructure to significantly enhance the performance of the conventional droop controllers [4].

The different methods that have been studied to address the issue of correct reactive power sharing among the distributed generators can be broadly divided into centralized, de-centralized and distributed control algorithms [24]. Micro-grid studies with centralized control systems have used particle swarm algorithms, mixed-integer linear algorithms and genetic algorithms but as mentioned these control systems require extensive communication systems and their performance starts to decrease as the number of distributed generators increase [8]. The decentralized control systems have also been extensively researched and mainly work on the principle of adding virtual impedances at the output of distributed generators. Each distributed generator senses the impedance of its micro-grid bus and changes its virtual impedance to properly match it [25]. Apart from the problem of increased voltage drop, this method does not perform well when large number of distributed generators involved, as this method assumes that each distributed generator feeder has a similar impedance characteristics [25]. This is not the case due to different ratings and size of the distributed generators, which results in a mismatch of impedance and the flow of circulating currents. Again, complex control and communication systems would be required to properly implement the decentralized control algorithms [22]. Therefore, as previously mentioned, the distributed control systems are best suited for the proper power sharing and control between the distributed generators. They require simple communication and control equipment, yet do not lose their operational functionality even when the micro-grid system is increased to incorporate large number of distributed generators [24]. 
Apart from consensus based algorithms, other distributed algorithms have also been studied. These include the 'Predictive control based algorithms', that uses a linearized model to predict the reactive power needed in the next time step and adjusts the output of distributed generators accordingly [26]; 'Agent based algorithms', that employs a 3 step communication system of a distributed generator with its neighbors and tries to implement a hierarchical control to keep the reactive power at an optimal value [27]; and 'Decomposition based algorithms', that decomposes the original optimization problem of reactive power into smaller problems, usually based on grouping of specific distributed generators and solving them until all of them converge [24]. From all the mentioned algorithms, the consensus based control is the simplest, requires the least communication resources and has the fastest convergence time [24].

\subsection{Thesis Objectives}

1. The purpose of this research is to implement the consensus based algorithm for the sharing of reactive loads among individual generators of the micro-grid clusters and to show that the respective control method ensures stable voltage levels at each micro-grid in different grid conditions.

2. To analyze the convergence analysis of the micro-grid cluster system under different communication topologies normally adopted in a typical micro-grid.

3. To study the effects of communication delays due to the different data transfer rates of different communication technologies.

4. To examine the effects of tripping of any of the distributed generator on the micro-grid cluster. 
5. To investigate the sensitivities of the micro-grid system due to the gain of consensus-droop controller and the effect of varying the load connected to the micro-grid.

\subsection{Outline of Thesis}

The remaining part of this thesis is organized in the following way:

- Chapter 2 covers the necessary background of the electrical system involved and the mathematical equations associated with them. It will also introduce the concept of graph theory and explain how the consensus algorithm works.

- Chapter 3 explains the modelling of a micro-grid clusters and the software used to perform the simulations. The details of the proposed controller and its mathematical dynamics will also be covered in this chapter.

- Chapter 4 presents the simulation results and discusses the performance of consensus based algorithm in each case simulated.

- Chapter 5 summarizes the complete thesis, discusses the effectiveness of consensus based algorithm and presents the directions for future work.

\subsection{Summary}

Reactive power sharing and voltage stability of micro-grids is still an area of concern as the conventional droop control does not work properly with distributed generators. As more independent micro-grids come in to service, this problem will become even more severe. The proposed consensus based algorithm has previously been successfully implemented in various fields but has yet to be tested in electrical 
power sharing control of generators. Therefore, this thesis will model a micro-grid cluster and simulate the consensus based algorithm for the sharing of reactive loads among individual generators in different grid conditions and show that this control could very well work in practical. 


\section{Chapter 2 Background}

\subsection{Introduction}

The background theory of the power system involved and the mathematical relationships involved will be explained in this chapter. To understand this thesis, it is important to know the exact nature of a micro-grid and how the micro-grid bus voltage can be stabilized with the control of the reactive power output of a generator. Furthermore, the droop equations have been derived and finally the basics of consensus algorithm has been explained to help us build towards the proposed controller.

\subsection{What is a Micro-Grid}

A micro-grid is usually defined as a group of various distributed generators, energy storage devices and loads that can function as a common power grid, working in islanded mode as well as when connected together to form a micro-grid cluster or with a large power network [28]. A typical scheme of a micro-grid has been shown in figure 2.1. The distributed generators can be of different types and ratings. Small rated distributed generators, typically in the range of $\mathrm{kW}$, can be owned by the utility customers themselves and can contribute to the generation requirements of the micro-grid as well. The distributed generators can conceptually be added and removed easily in a 'Plug and Play' model. This means that the individual distributed generators are able to add or disconnect themselves from the micro-grid without altering the control or protection system of the generators connected to the grid [29]. It is important that for the micro-grid to perform as a large single electrical 
system that may be able to maintain the electrical grid parameters throughout the micro-grid, it is necessary that all the distributed generators, regardless of the nature of the primary energy source, use power inverters to integrate themselves, providing the overall micro-grid with the essential flexibility and control [30].

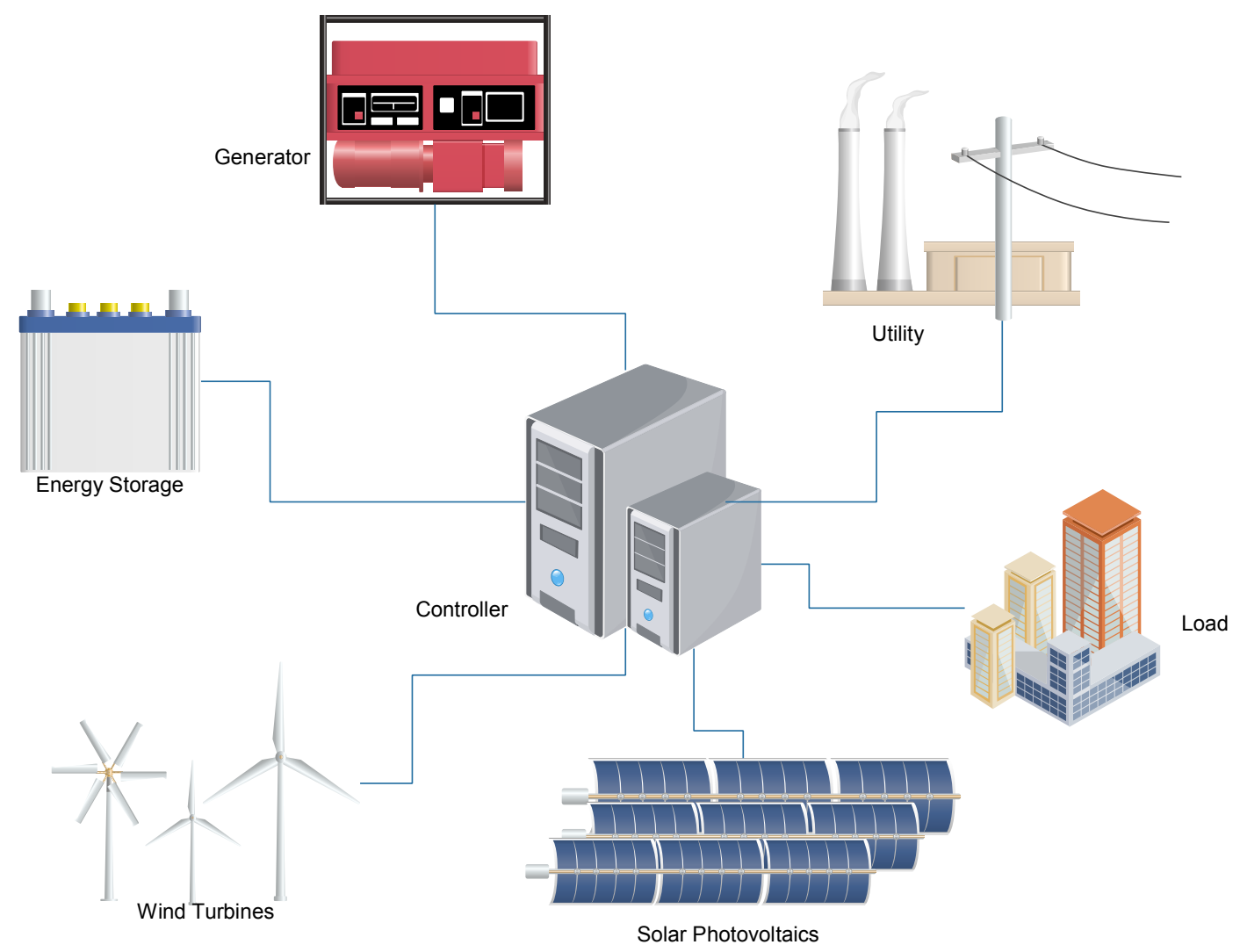

Figure 2.1: Typical scheme of a Micro-grid

Apart from the integration and interfacing issues of distributed generators in microgrids and the inevitable protection problems, the main concerns of the micro-grid are the control of power flow, voltage stability and the uniform sharing of active power and reactive power between the distributed generators. 
The control of micro-grids are currently based on the conventional droop control of generators but has been enhanced into a 3 level hierarchal control [31]. The primary control of the micro-grid provides the distributed generators with an overall set point to keep the micro-grid stable, within the tolerance band, at the required rated voltage and frequency. The secondary control is to cater for the small deviations of the primary controller from the true value and works slower than the primary control. The tertiary control is the slowest and determines the flow of active and reactive power between the micro-grid and the other connected micro-grid or the main grid [31, 32]. This is also explained in figure 2.2.

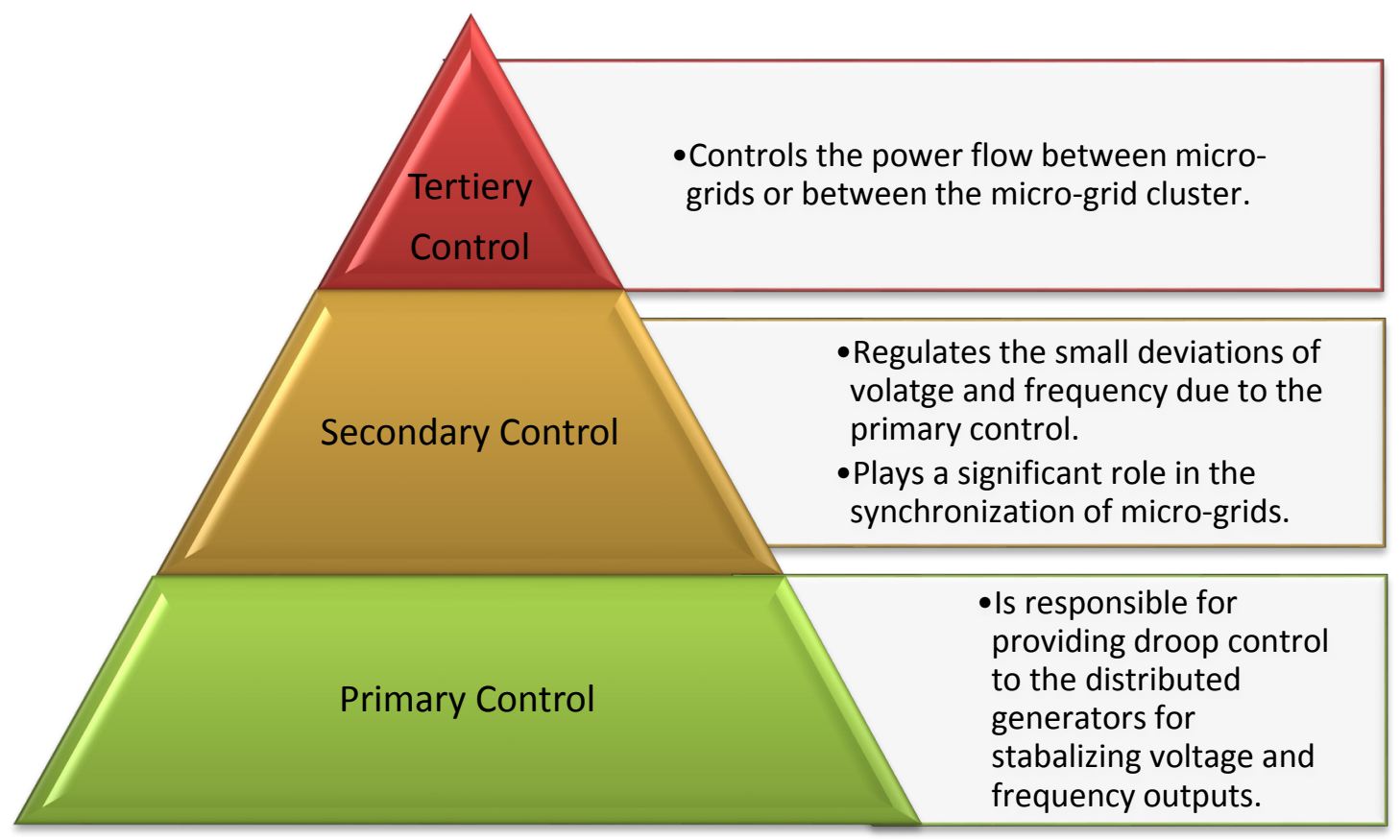

Figure 2.2: Hierarchal control of a Micro-grid 


\subsection{Relationship between Reactive Power and Voltage Stabilization in Micro- Grids}

Reactive power is an important factor in AC power systems. It is usually mentioned as the 'imaginary' power in textbooks but is the effect due to the phase difference between the voltage and current waveforms in an AC circuit. The more the phase difference between the two, the more reactive power is produced. The function of reactive power is to produce and maintain the electric and magnetic fields in $A C$ electrical machines and equipment.

Another vital role of reactive power is to establish and maintain the necessary voltage levels of the $A C$ electrical system as they are both directly proportional to one another. The stability of voltage level is essential for the electrical system to work properly since the voltage level ensures that the 'active' power flows easily from the generators to the loads. When the reactive power is not sufficient, the voltage 'sags' and there is not enough push available to transfer active power. If the voltage is low, then the electrical equipment performs poorly, for example, the dimming of light bulbs and the overheating of induction motors are examples of such. If the voltage level is too high, then the electrical equipment can burn out, be permanently damaged or have their insulation levels degraded. Therefore, the voltage level of an electrical system recommended is within $\pm 5 \%$ of the rated voltage of the system.

Voltage stability is one of the key reasons resulting in major blackouts of power grids throughout the world. Insufficient control of reactive power produced by network generators lead to a system voltage failure that result in the tripping of all the generating sources, transmission lines and distribution feeders. In US, this has 
happened in the black outs of July '96 and August '96. Prabha Kundur, in his book writes that, "Voltage stability is the ability of a power system to maintain steady acceptable voltages at all buses in the system under normal operating conditions and after being subjected to a disturbance. A system enters a state of voltage instability when a disturbance, increase in load demand, or change in system condition causes a progressive and uncontrollable drop in voltage. The main factor causing instability is the inability of the power system to meet the demand for reactive power. " [18]

\subsection{Derivation of Micro-Grid P-f and Q-V Equations}

To understand the electrical fundamentals of the behavior of micro-grid voltage and frequency, assume a simple electrical branch of a transmission line through which active and reactive power both are flowing, as seen in figure 2.3.

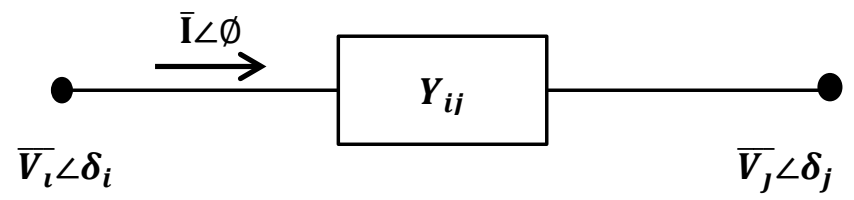

Figure 2.3: A simple current carrying electrical transmission line.

Then the total power, active and reactive power, flowing through the line is given by the following formula:

$$
S=P+j Q=\Delta V \cdot I^{*}
$$




$$
\begin{gathered}
S_{i j}=V_{i \cdot}\left(\sum_{j=1}^{N} Y_{i j} \cdot V_{j}\right)^{*} \\
S_{i j}=V_{i} \angle \delta_{i \cdot} \sum_{j=1}^{N}\left(G_{i j}+j B_{i j}\right)^{*} \cdot\left(V_{j} \angle \delta_{j}\right)^{*} \\
S_{i j}=\sum_{j=1}^{N} V_{i} \angle \delta_{i \cdot}\left(G_{i j}-j B_{i j}\right) \cdot\left(V_{j} \angle-\delta_{j}\right) \\
S_{i j}=\sum_{j=1}^{N} V_{i} V_{j} \angle\left(\delta_{i}-\delta_{j}\right) \cdot\left(G_{i j}-j B_{i j}\right) \\
S_{i j}=\sum_{j=1}^{N} V_{i} V_{j}\left(\cos \delta_{i j}-\mathrm{j} \sin \delta_{i j}\right) \cdot\left(G_{i j}-j B_{i j}\right)
\end{gathered}
$$

Where, $\delta_{i j}=\delta_{i}-\delta_{j}$

Therefore, the real part becomes the active power:

$$
P_{i j}=\sum_{j=1}^{N} V_{i} V_{j}\left(G_{i j} \cos \delta_{i j}+B_{i j} \sin \delta_{i j}\right)
$$

The imaginary part becomes the reactive power:

$$
Q_{i j}=\sum_{j=1}^{N} V_{i} V_{j}\left(G_{i j} \cos \delta_{i j}-B_{i j} \sin \delta_{i j}\right)
$$

For small angle deviations, the assumptions that $\sin \delta \cong \delta$ and $\cos \delta \cong 1$ holds true and therefore the above equations of active power and reactive power can be rearranged into: 


$$
\begin{aligned}
& \delta_{i j} \cong \frac{P_{i j}}{V_{i} V_{j} B_{i j}}-\frac{G_{i j}}{B_{i j}} \\
& V_{i} V_{j} \cong \frac{Q_{i j}}{\left(G_{i j}-B_{i j} \cdot \delta_{i j}\right)}
\end{aligned}
$$

Hence, it can be easily seen that the flow of active power depends upon the magnitude of the 'power angle'. This power angle when changes with respect to time and is divided by $2 \pi$ becomes frequency. The reactive power depends on the magnitude of the voltages. The linear relationship between the frequency and active power is called ' $P$ - $f$ droop' and the linear relationship between voltage and reactive power is called ' $Q-V$ droop'.

\subsection{Droop Control in Generators}

Rotating machines are an integral part of AC electrical systems and can be power sources, like synchronous generators, or can be an electric load, like induction motors and synchronous motors. For a smooth operation, they require the system frequency to be fixed at a constant frequency but any load increase on the network results in an increase of load torque (or power) requirements that result in the decrease in the rotational speed of the synchronous generator prime movers, the mechanical power source machines running the generators. This decrease in speed results in a decrease in the generator frequency and in turn the network frequency.

To correct this decrease in frequency, the generator's prime mover increases its fuel throttle and increases its speed by a fixed constant value, called the 'Speed Droop'. This proportional value is also usually referred to as the P-f droop control. If the system frequency increases, then the generator prime movers decrease their speed 
in the same proportional droop value to keep the system frequency steady. The following is the formula used to calculate the initial droop control setting of the generator:

$$
D_{s}=\frac{S_{n l}-S_{f l}}{S_{f l}} \times 100 \%
$$

Where, $\quad D_{S}=$ Generator speed droop capability

$S_{n l}=$ No load speed of the generator

$S_{f l}=$ Full load speed of the generator

Since the electrical system is more concerned with power and frequency of the generator, this relationship can also be written as following [33], and is also shown in figure 2.4:

$$
f-f_{0}=-S p \times\left(P-P_{0}\right)
$$

Where, $\quad f=$ Frequency of the generator

$f_{0}=$ Nominal frequency

$S p=$ Slope of the P-f curve

$P=$ Active power of the generator

$P_{0}=$ Nominal active power 


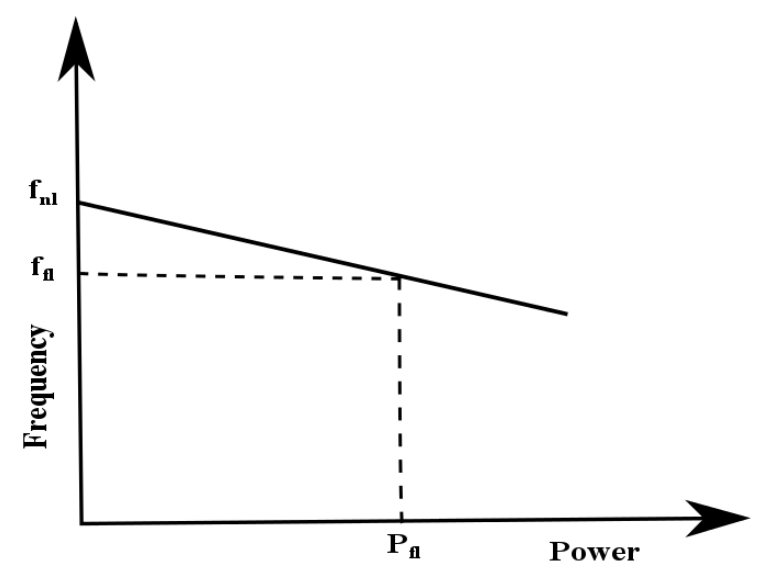

Figure 2.4: Power-Frequency Droop Curve

The same relationship holds between the reactive power output of the generator and the voltage at its output terminals. Reactive power is consumed by inductive load and hence any addition of reactive load on the grid would decrease the available reactive power necessary to maintain the voltage. The generators will then need to increase the output reactive power generated by them to bring the voltage back to the desired value.

The formula used to calculate the initial voltage droop control setting of the generator is:

$$
D_{v}=\frac{V_{n l}-V_{f l}}{V_{f l}} \times 100 \%
$$

Where, $\quad D_{v}=$ Generator voltage droop capability

$V_{n l}=$ No load voltage of the generator

$V_{f l}=$ Full load voltage of the generator 
As in the case of the relationship between reactive power and voltage of the generator is similar and can also be written as [33] and is similarly shown in figure 2.5:

$$
V-V_{0}=-S q \times\left(Q-Q_{0}\right)
$$

Where, $\quad V=$ Voltage of the generator

$V_{0}=$ Nominal voltage

$S q=S l o p e$ of the Q-V curve

$Q=$ Reactive power of the generator

$Q_{0}=$ Nominal reactive power

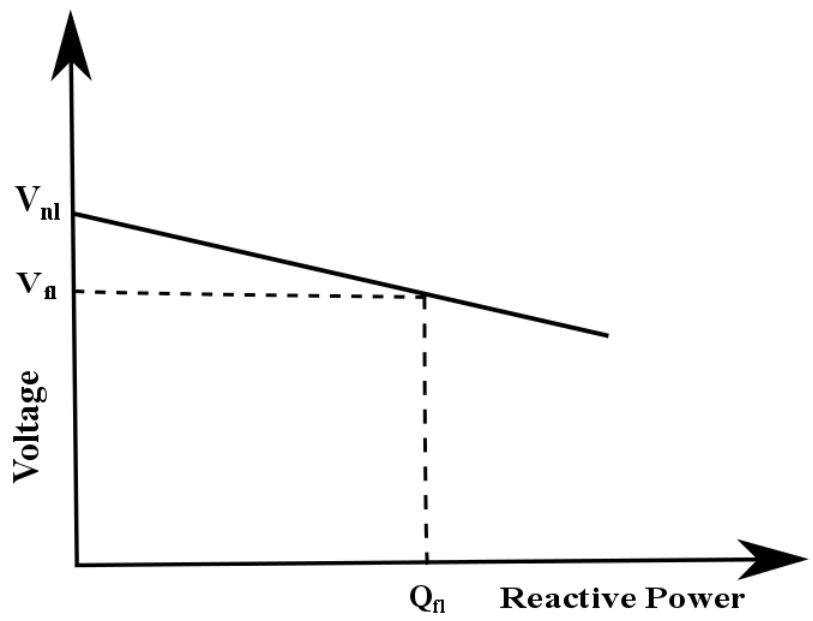

Figure 2.5: Reactive Power- Voltage Droop Curve 


\subsection{Graph Theory and Consensus Algorithm}

To understand consensus based algorithm, it is first necessary to review the basic mathematics of graph theory. Consider a system with several independent units in the following dynamic system:

$$
\dot{x}_{i}=u_{i}
$$

Where, $\quad \dot{x}_{i}=$ System of element $x_{i}$

$u_{i}=$ Output of the system

All units want to achieve consensus among themselves using communication only with their neighbors within a graph stated as:

$$
G=\left(V_{G}, E_{G}\right)
$$

where, $V_{\mathrm{G}}$ is a set of finite and non-empty elements called 'nodes' or 'vertices' and $\mathrm{E}_{\mathrm{G}}$ is a set of unordered two elements of vertices, $\mathrm{V}_{\mathrm{G}}$, called 'edges'.

Graph is called 'simple' when it is undirected, unweighted, and does not have more than one edges or loops [4]. For the rest of our study, we will consider the term graph to be as a simple graph. A connected graph must have a direct path between at least two distinct nodes of the graph.

The communication topology of the simple graph with $n$ vertices is represented by a $n \times n$ matrix called the 'adjacency matrix', $\mathbf{A}_{G}$. The off-diagonal entry of the adjacency matrix is to determine if there is a direct path from node $i$ to node $j$, that is: $a_{i j}=1$ if $E_{G}(i, j)=1$. Otherwise $a_{i j}=0$, including the diagonal terms. The 
adjacency matrix is symmetric whenever there is an undirected graph under consideration.

The number of edges of each node of the graph is denoted by another $n \times n$ matrix, called the 'degree matrix', $\mathbf{D}_{\mathrm{G}}$. The degree matrix is a diagonal matrix in which the diagonal entries $d_{i=j}$ represents the number of edges of the node $i$. All other entries of the matrix are zero.

Together the adjacency matrix and the degree matrix help to find out the 'Laplacian matrix', $\mathbf{L}_{G}$, of the graph. The Laplacian matrix represents the complete communication topology and the connectivity between all the nodes of the graph and is defined as:

$$
\boldsymbol{L}_{G}=\boldsymbol{D}_{G}-\boldsymbol{A}_{G}
$$

The entries of the Laplacian matrix can also be directly calculated using the following relationship with the adjacency matrix:

$$
\begin{gathered}
l_{i j}=-a_{i j} \text { when } i \neq j \\
l_{i j}=\sum_{i=1}^{n} a_{i j} \text { when } i=j
\end{gathered}
$$

The consensus based algorithm can now be defined as the following [7]:

$$
\dot{x}_{l}(t)=\sum_{j \neq i}^{n} a_{i j}\left(x_{j}(t)-x_{i}(t)\right)
$$

The above equation ensures that all the nodes in the graph converge to a collective agreement asymptotically, using only neighbor node communications. The above equation is also used to measure the 'diffusion' movement of gas particles based on 
their densities [4]. The same way, the spread of information can be studied using the above equation under consensus based algorithm.

The value of the consensus that is achieved is found when the time, $t$, goes to infinity, that is:

$$
\lim _{t \rightarrow \infty} x_{i}(t)=\lim _{t \rightarrow \infty} x_{j}(t)=\frac{1}{n} \sum_{i}^{n} x_{i}(0)
$$

This shows that all the nodes asymptotically reach the average value of all the initial states of the nodes when consensus is achieved.

The equation stated for the consensus algorithm earlier is for continuous time and can be converted to a discrete time equation as [7]:

$$
x_{i}(k+1)=x_{i}(k)+\in \sum_{i \neq j}^{n} a_{i j}\left(x_{j}(k)-x_{i}(k)\right)
$$

Where is the $\epsilon=$ Time step used for the iterations and should be greater than 0 .

\subsection{Summary}

This chapter discussed all the basics and background knowledge about micro-grids and the issues associated with them. It explained how the control of reactive power of a generator can easily help control the micro-grid bus voltage and tells us how the droop control works. The overview of graph theory and consensus algorithm is then explained. 


\section{Chapter 3 Modelling and Control of Micro-Grid Clusters}

\subsection{Introduction}

It is important to properly define and model the micro-grid cluster to effectively simulate the real case scenario and implement the consensus algorithm. In this chapter, we will discuss the typical power flow software solutions and the issues associated with modelling distributed generation in them. The modelling done in this thesis will then be explained and it will be investigated how accurately the model represents the actual micro-grid. The software that has been used for the simulations and the final model of micro-grid clusters will also be described along with the workings of the proposed controller.

\subsection{Describing Micro-Grids in Commercial Power Flow Software}

There are several commercial software available in the market that can model and perform electrical power analysis including load flow study, transient stability, fault analysis and power distribution. Each one of them has their own advantages and disadvantages in terms of the functionality and the available electrical control blocks but nearly all of them lack a dedicated description and necessary tools to model distributed generators. This is mainly because the commercial integration and network penetration of distributed generators is still relatively low as compared to 
the conventional synchronous generators. Also, the type and internal functionality and dynamics of the distributed generators can vary depending upon the nature of the renewable energy source being used and the control technology involved.

Another issue commonly faced is that, these software assume that the overall frequency of the electrical system remains constant [34]. This can be a significant problem when working on the dynamics of active power flow in micro-grids, since due to the inherent size and nature of the micro-grids, they experience significant frequency changes with changes in large-loads. The issue has been usually addressed by researchers by putting up an additional P-f droop characteristics in the frequency control of the micro-grid generators themselves, mimicking a change on the micro-grid bus based on the variations of large loads. Since this study is primarily based on the voltage stabilization of the micro-grids using consensus algorithm, we do not have to worry much about the frequency control and therefore, we have taken the same assumption of constant frequency in our simulation modelling.

Distributed generation might be of different types and may have different internal functionalities, but in overall, they still follow the conventional electrical power flow dynamics and hierarchical droop equations, when they are connected together to form micro-grids. This is because the loads as well as the distribution and transmission infrastructure still works on the conventional droop system. The most convenient and cost effective solution for the integration of micro-grids to the present power systems is to make the new distributed generation behave like a conventional synchronous generator. And hence, from the perspective of micro-grid simulation and load flow analysis, the distributed generators can be effectively assumed as synchronous generators without any major deviations from the actual behavior. This is precisely what has been assumed for the simulation modelling used for this thesis. 


\subsection{Modeling of a Micro-Grid}

The software solution used in this study is the Power System Computer Aided Design (PSCAD). This software is very popular among researchers in electrical power as it offers a wide range of controls, simulation features and analytical tools. The software has a large library of electrical components, is flexible to modification of any of the tool functionality and is user friendly.

As mentioned in the previous section, the distributed generators have been modelled using a 3-phase synchronous generator that externally takes in feedback for the magnitude of voltage, the phase angle at which the generator should operate and the frequency at which the generator should give its output. The frequency and phase angles are used for the control of the active power output of the generator while the voltage magnitude caters for the reactive power. Since this study is only concerned with the proper regulation of reactive power and stabilization of the micro-grid bus voltage, the frequency input has been fixed at $60 \mathrm{~Hz}$. The P-f droop control is used to provide the respective generator with the required phase angle necessary for the generator's fixed active power dispatch. 


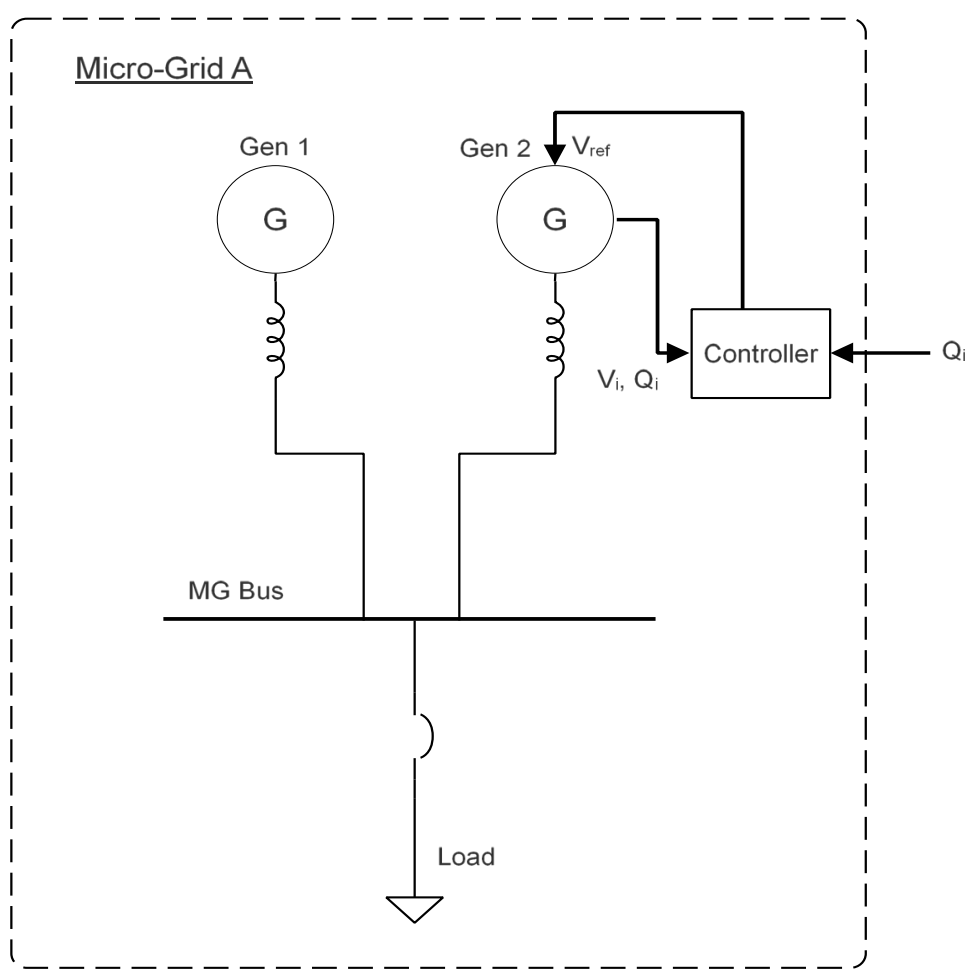

Figure 3.1: Model of a Micro-Grid under consensus control

In figure 3.1, the micro-grid has been modelled with two generators connected to a single common micro-grid bus that provides power to the connected loads. Each generator is connected to a micro-grid bus through its output impedance and has a unique power rating.

As the generators of the micro-grid modelled are similar but unique, so are the loads connected to the micro-grid. Three different impedance loads have been added to each micro-grid. The largest of the three is the base load and remains fixed throughout the simulations. The other two loads are switched on the micro-grid at different times of the simulation depending upon the simulation case being studied. Each micro-grid can easily supply power to and cater for all its connected loads when working in islanded mode. Table 3.1 summarizes the values of the electrical parameters used for modelling a micro-grid: 
Table 3.1: Electrical Parameters used for to model the micro-grids.

\begin{tabular}{|l|c|c|c|c|c|c|}
\hline \multicolumn{1}{|c|}{ Parameters } & \multicolumn{2}{c|}{ MG-A } & \multicolumn{2}{c|}{ MG-B } & \multicolumn{2}{c|}{ MG-C } \\
\hline & Gen 1 & Gen 2 & Gen 3 & Gen 4 & Gen 5 & Gen 6 \\
\hline Power Rating (MVA) & 800 & 700 & 700 & 500 & 650 & 800 \\
\hline Voltage Rating (KV) & \multicolumn{7}{c|}{230} \\
\hline Base Frequency (Hz) & \multicolumn{7}{c|}{60} \\
\hline Generator Inductance (H) & 0.01 & 0.05 & 0.021 & 0.025 & 0.012 & 0.042 \\
\hline Active Power Reference (MW) & - & 550 & 600 & 350 & 475 & 650 \\
\hline Generator Reactive Droop & 0.25 & 0.231 & 0.263 & 0.258 & 0.275 & 0.266 \\
\hline Voltage Time Constant (s) & \multicolumn{7}{|c|}{0.05} \\
\hline Base Load (MW/MVAR) & $900 / 390$ & $600 / 390$ & $1050 / 345$ \\
\hline Variable Load (MW/MVAR) & \multicolumn{7}{|c|}{$225 / 135$} & \multicolumn{7}{|c|}{$300 / 105$} & $285 / 120$ \\
\hline
\end{tabular}

\subsection{Micro-Grid Clusters}

To effectively demonstrate the performance of the consensus algorithm, 3 microgrids have been combined together to form a micro-grid clusters. Bus-ties have been used to connect the micro-grids through line impedances on both sides of the bustie. Again, the impedances have been kept unique to model for the different lengths of the connecting lines in between the micro-grid. The bus-ties can be opened or closed at any time with proper synchronization of one micro-grid to another.

When the bus-ties are open, each micro-grid works in an island mode with one generator behaving as an isochronous generator, meaning that the function of that generator is to maintain the micro-grid voltage and frequency. For that purpose, it can change its output active and reactive powers as per the need of the micro-grid. The other generator works on droop mode giving a fixed output of active and reactive powers.

The consensus based control algorithm has been designed in such a way that the consensus control for the micro-grids involved, only comes into action when the bus-tie closes in between the two micro-grids, as shown in figure 3.2. In that case, all 
the isochronous generators except one, automatically shift to a droop mode and start working together with the other generators in consensus mode. It is important to keep at least one generator in isochronous mode so that it can provide a voltage reference point for the other generators to follow during the consensus.

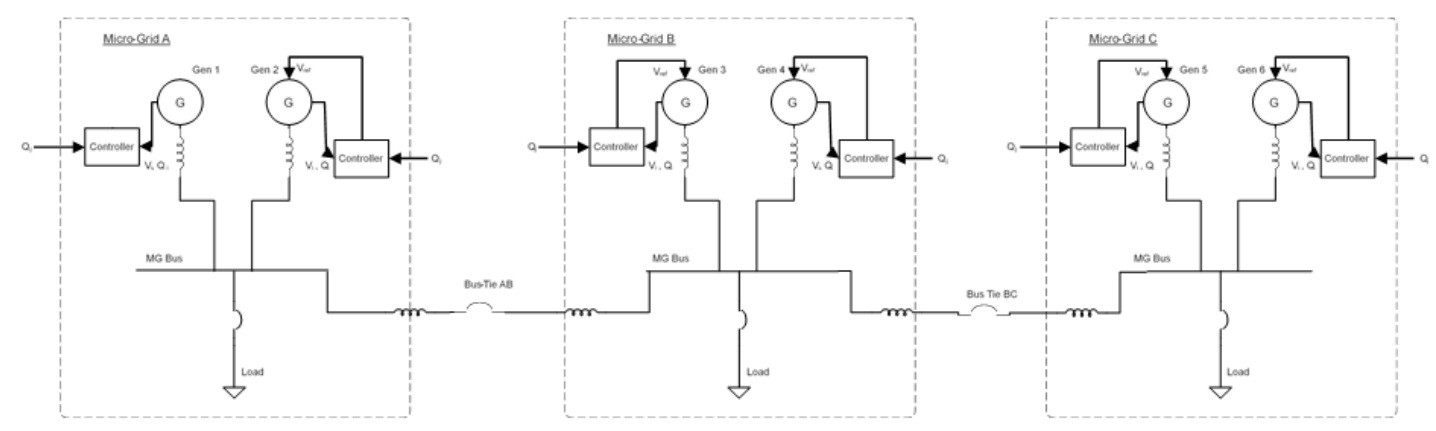

Figure 3.2: Single line diagram for the Micro-grid clusters under consensus control

\subsection{Proposed Consensus Based Droop Controller for Voltage Stabilization}

The control algorithm proposed by this study is a combination of both the conventional droop and the consensus based algorithm. The conventional droop helps the distributed generator to behave like a synchronous generator and regulate the generator output in a controlled manner. The consensus algorithm helps to provide each generator with the desired set point that it should achieve by regulating its output.

As the primary purpose of this research is to demonstrate voltage stability, the frequency of the micro-grid is assumed to be fixed at its nominal value and the real power portions generated by the distributed generators are modelled based on the conventional droop. Since the data transfer through communication channels is always in discrete packets and takes time to travel from the source to its destination, 
we will be using the discrete time model of the consensus algorithm and modifying it by incorporating the droop control.

As explained earlier, for voltage stability of micro-grids, the parameter that needs to be regulated is the reactive power of each generator. Hence our $x$ in study is the output reactive power given by each generator.

Since the software, PSCAD, used for this research requires the feedback and the set point of the generator to be given in terms of the magnitude of voltage, we will use the consensus algorithm to find the desired value of the reactive power for each generator and we will use the droop of the generator to convert it to the voltage set point feedback of the respective generator. Following is the equation that defines the proposed consensus algorithm with droop control and is also explained through a control diagram in figure 3.3:

$$
V_{i}(k+1)=V_{i}(k)+S q_{i}\left[\in \sum_{i \neq j}^{n} a_{i j}\left(Q_{j}(k)-Q_{i}(k)\right)\right]
$$




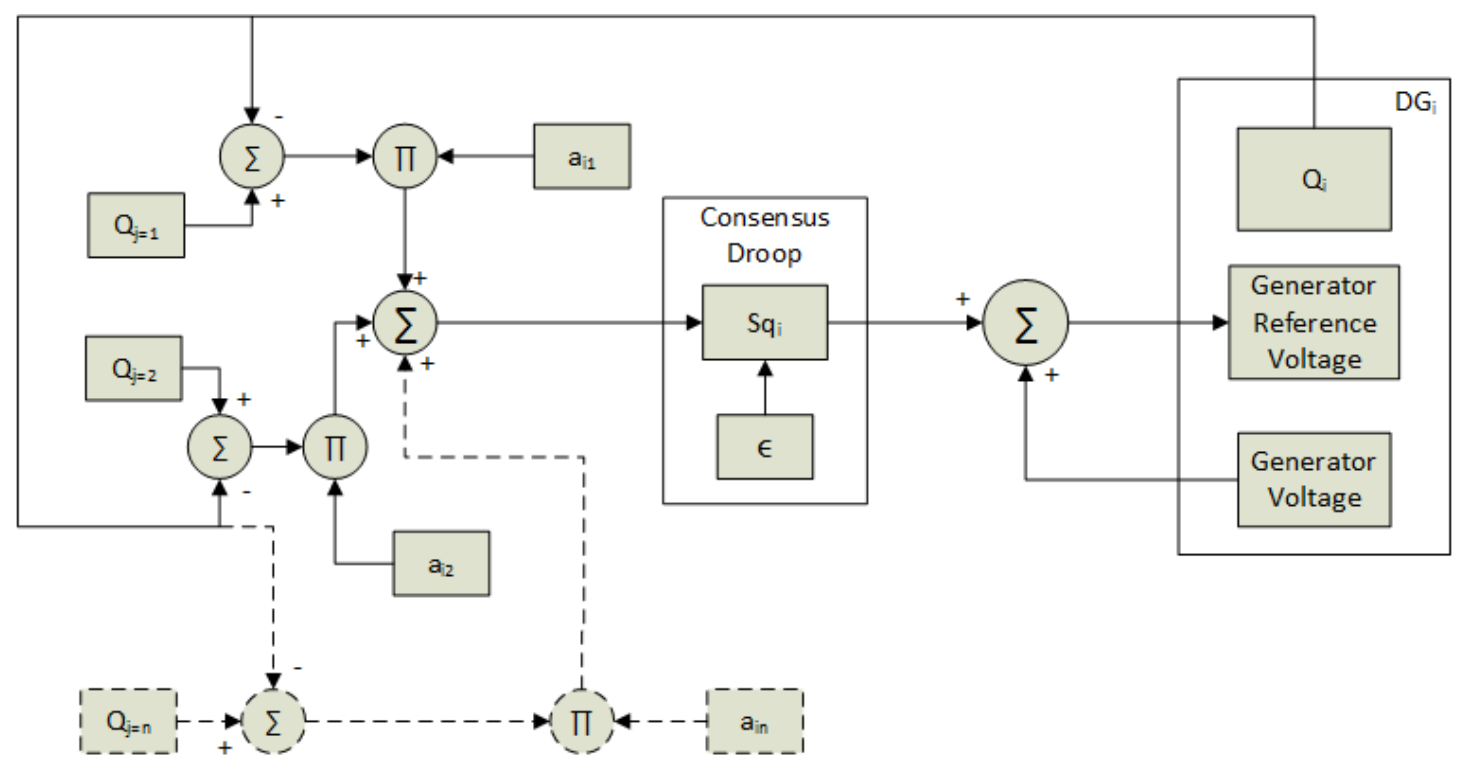

Figure 3.3: Block diagram of Consensus Control

\subsection{Summary}

This chapter has explained the applications of various power system software solutions and described the limitations faced in them. PSCAD software was found to be the most suitable software for this study. The description of the micro-grid modelled as well as the entire micro-grid cluster has been explained along with the operation modes of the generators when the micro-grids are working in an islanded mode or when they are connected together along with the working of the proposed consensus droop control for the reactive power sharing between the individual distributed generators of the micro-grid. 


\section{Chapter 4 Case Studies}

\subsection{Introduction}

This chapter shows the simulations case studies under droop control, consensus based control, simulations with different communication topologies. Variable inductive load is added at 10 seconds, 20 seconds and 30 seconds at two separate micro-grids together to see the effect of bus voltages due to load variations.

\subsection{Base Case}

In the base case, all the micro-grids are connected together through the bus-ties but there is no communication between the distributed generators. Generator 1 of micro-grid $A$ serves as the isochronous generator and the rest of the generators are controlled through the conventional droop.

Figure 4.1 shows the simulation of the base case. As expected, there is no control of the bus voltages at micro-grid B or micro-grid C since the generators in these microgrids are severely affected by the inductive load that is connected to the overall grid. Micro-grid $C$ reaches to 1.2 times and micro-grid $B$ reaches to 1.14 times the rated voltage when the inductive load on the network is low as compared to their own micro-grids. Micro-grid A remains constant at slightly below the rated voltage since the isochronous generator, that is the generator that regulates the system frequency and voltage, is connected to that micro-grid which helps maintain the micro-grid voltage but not necessarily providing the required share of the reactive power. 

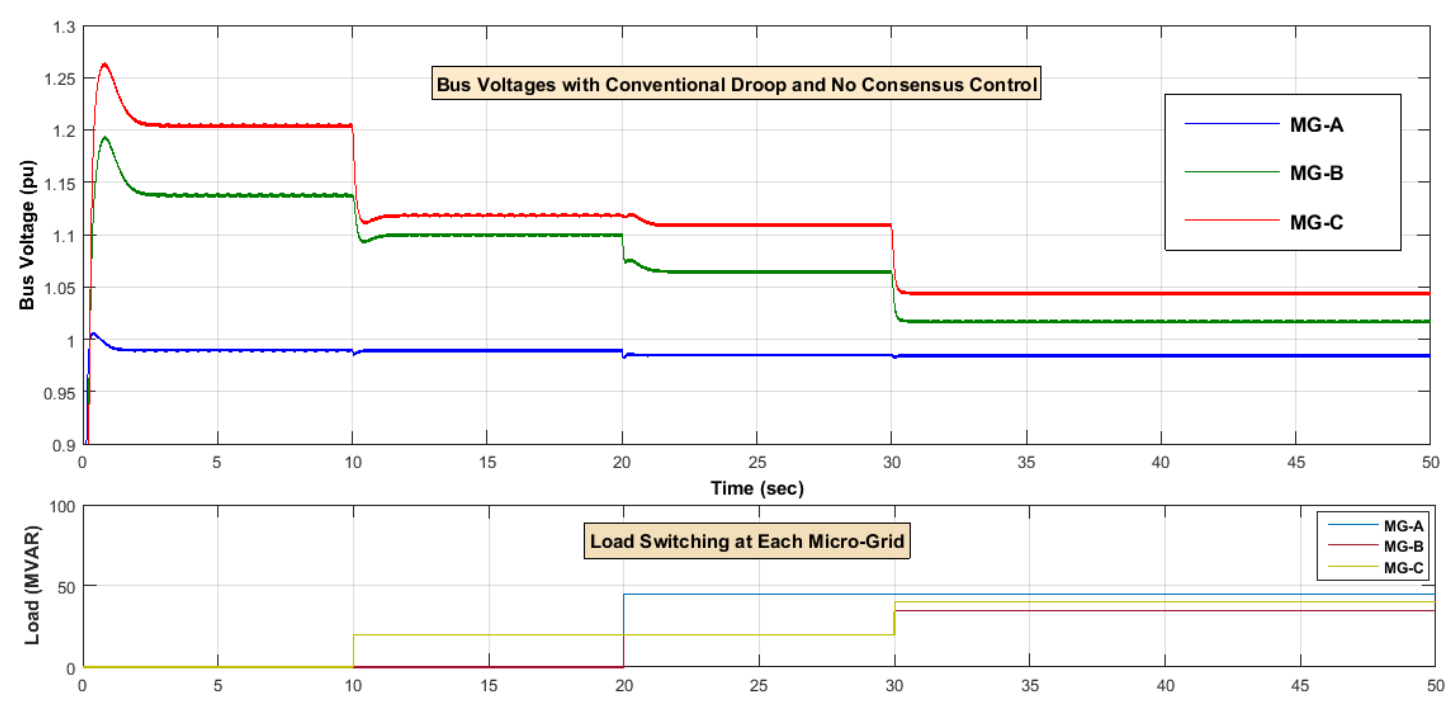

Figure 4.1: Comparison of micro-grid voltages with no consensus control

\subsection{Consensus Control with Different Communication Topologies}

The following simulation results are under the control of consensus algorithm. Since the consensus control is dependent on the degree of connectivity between its agents and hence the communication topology that exists between the generators. This thesis is going to demonstrate the three common communication topologies used in a distributed network and discuss the merits of each network. 


\subsubsection{Case I- Daisy Chain Topology}

The daisy chain topology is the most simple communication approach and requires each generator to be connected in series with their neighboring generators. Each generator is connected to two generators except the generators at the two ends of the communication line, which are connected with only one generator as seen in figure 4.2 .

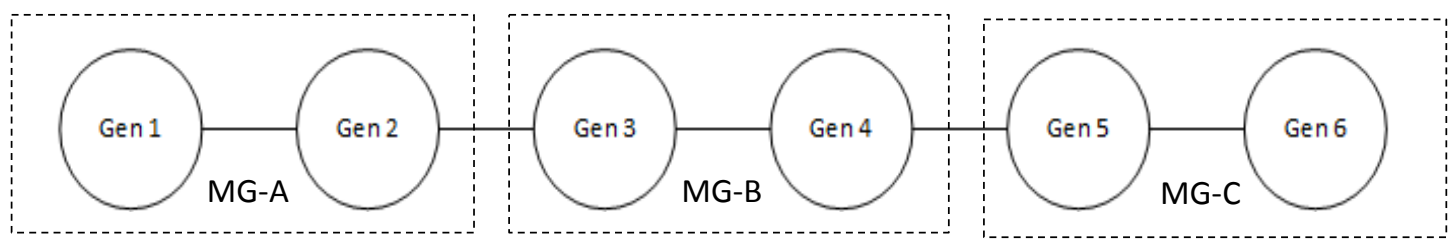

Figure 4.2: Daisy Chain Topology
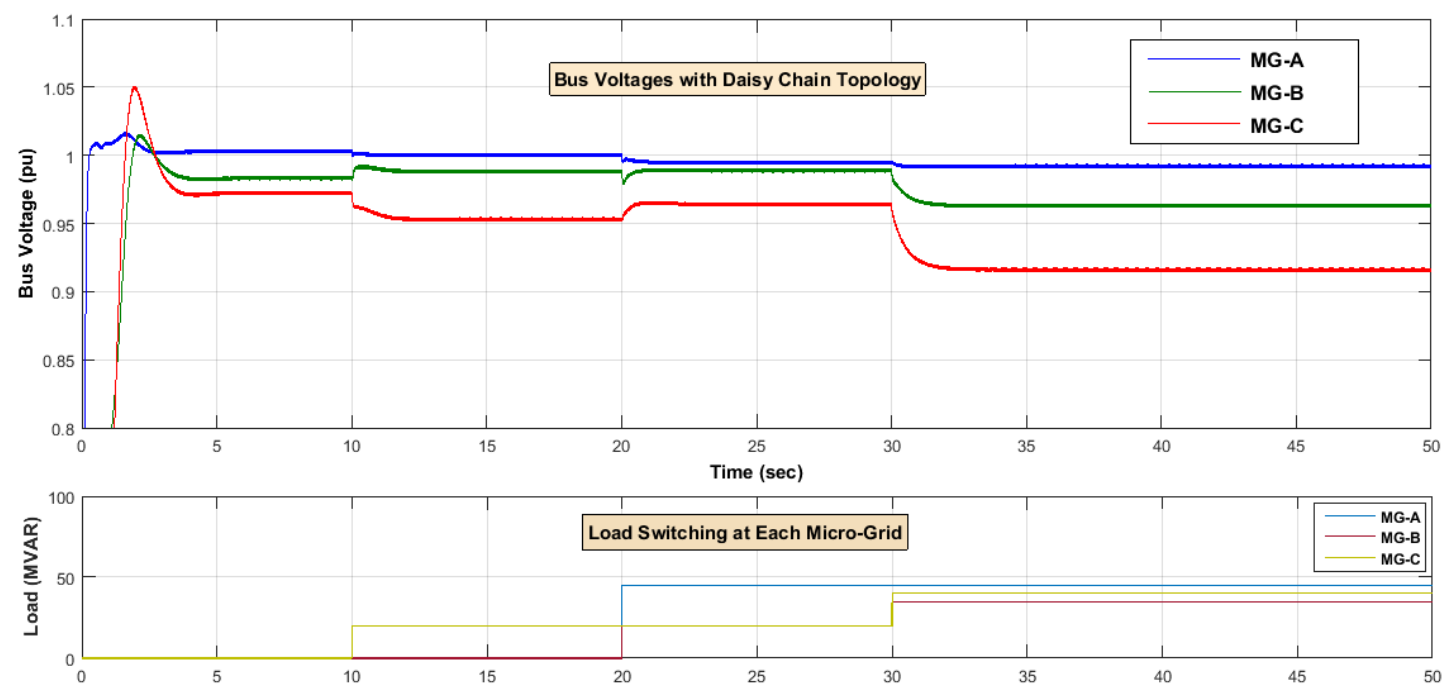

Figure 4.3: Comparison of Bus voltages with Daisy Chain communication topology 
The figure 4.3 shows the simulation results. The voltage profile is much better and brings $15.5 \%$ improvement in the voltage of micro-grid $B$ and $23.5 \%$ improvement in the voltage of micro-grid $\mathrm{C}$ as compared to the voltages with no consensus. Microgrid A stays close to the rated voltage due to the isochronous generator connected to it. Micro-grid B does experience voltage variations due to load addition but still remains within the $\pm 5 \%$ tolerance band. Micro-grid C experiences the most voltage drop due to the inductive load and is not able to regulate its bus voltage back to the normal range. This is primarily because of generator 6 , which is the last generator in the communication line. Generator 6 is only connected to only one generator and that too of the same micro-grid and hence the addition of load on the overall network is not properly recognized and regulated which results in a much higher voltage drop. Micro-grid C is also the grid furthest from the isochronous generator maintaining the overall voltage of the grid and providing a reference point for others. Yet it can be seen that the consensus algorithm is fast responding and the network of micro-grids reaches a consensus within 3.0 seconds of any load disturbance. 


\subsubsection{Case II- Loop Topology}

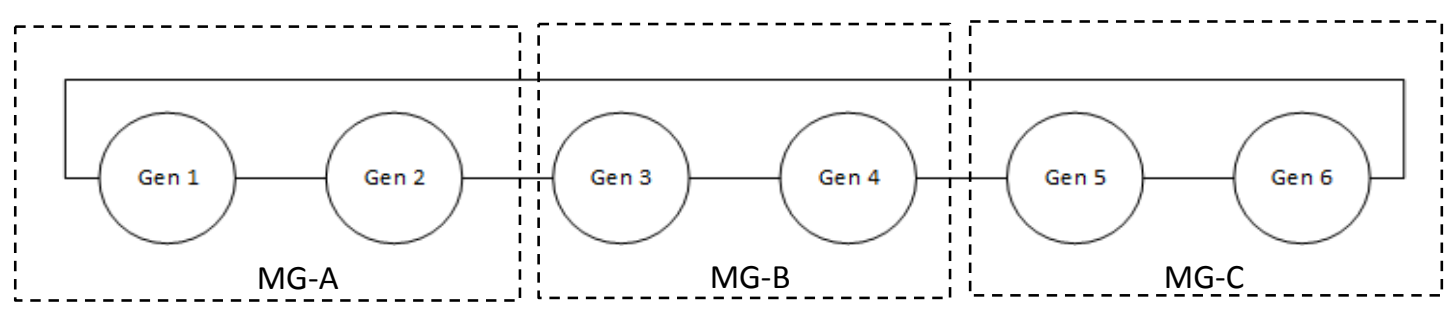

Figure 4.4: Loop Topology
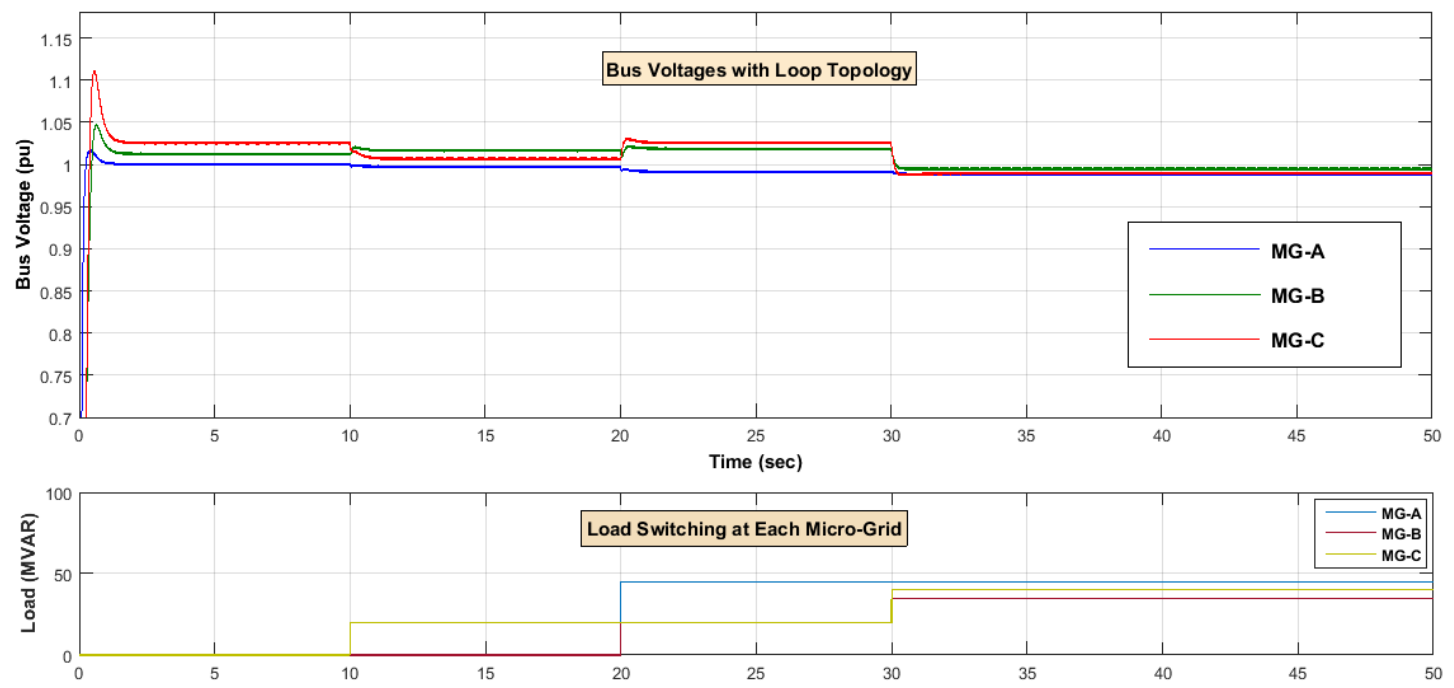

Figure 4.5: Comparison of Bus Voltages when all generators connected in a Loop

The drawbacks of the daisy chain topology are effectively addressed by the loop communication topology. The only difference between the two communication arrangements is that in loop communication, the last generator in the communication line, generator 6 of micro-grid in our case, is connected back to generator 1 of micro grid $A$, as shown in figure 4.4 . 
The simulation results, in figure 4.5 , are perfect and show a high coordination between the three micro-grids. Even though the inductive load additions have voltage variations on each micro-grid but the bus voltages remain within the voltage tolerance band. The time taken by the grid to reach a consensus decreases to 1.5 seconds. This is mainly due to the improvement in the connectivity and coordination among the generators as each generator has two generators to align itself with.

\subsubsection{Case III- Star Topology}

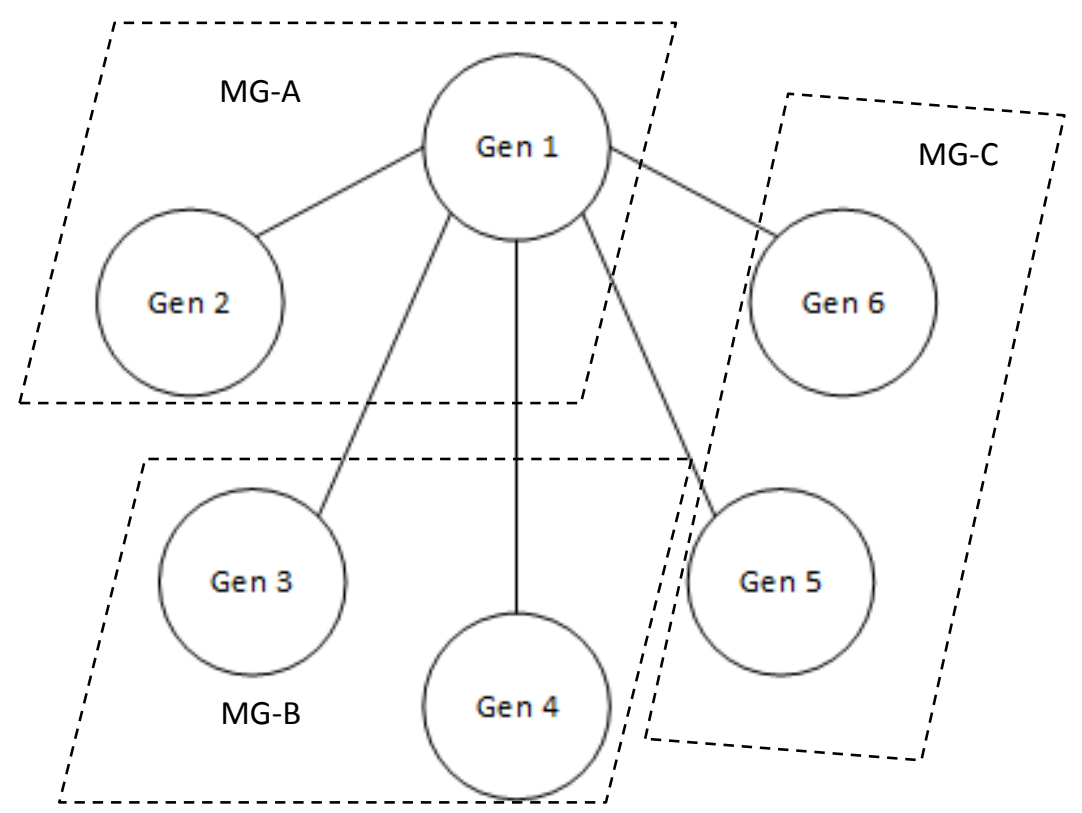

Figure 4.6: Star Topology 

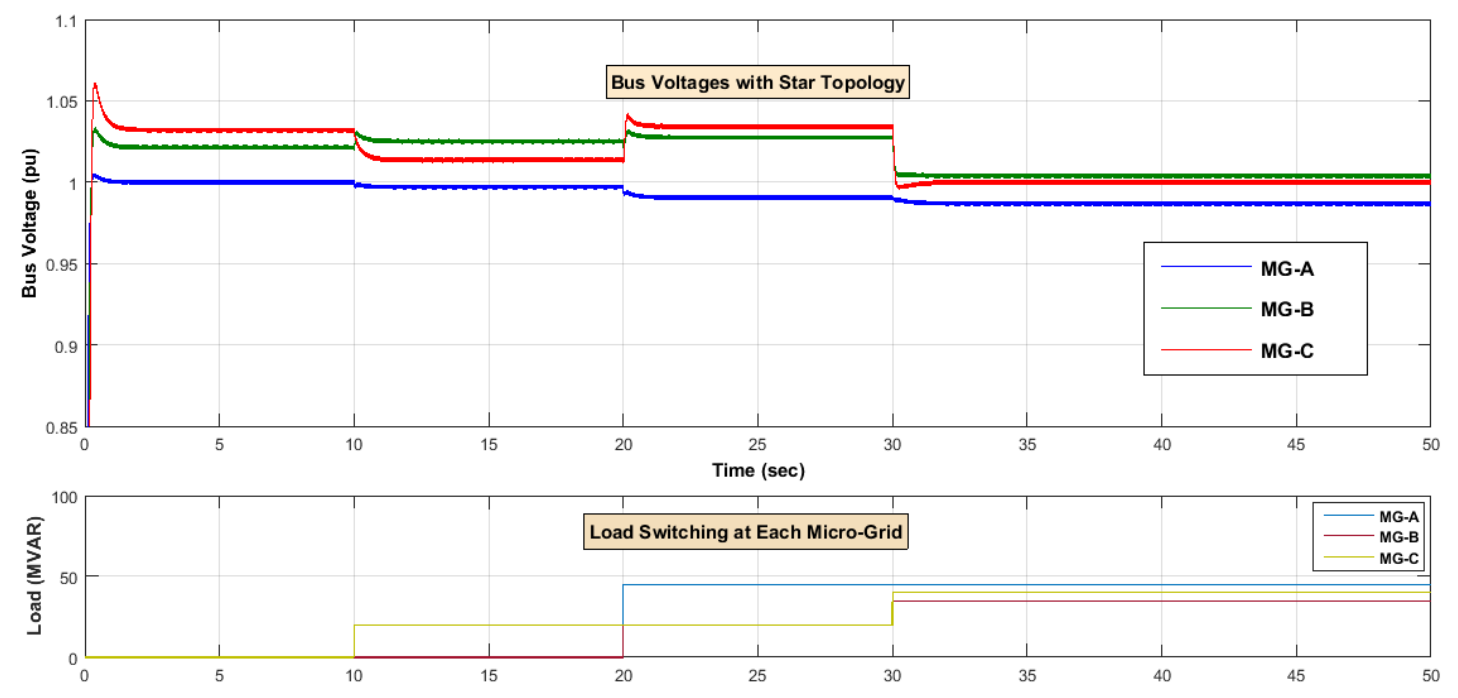

Figure 4.7: Bus Voltages when all generators connected in star topology

As generator 1 of micro-grid A provides a reference voltage for all other generators to follow, another possible communication arrangement could be such that each generator is connected just to generator 1 and tries to follow its path, as depicted in figure 4.6. The simulation results in figure 4.7 shows that this topology can also work even though the consensus time for this is 2 seconds, which is longer than the looped communication network.

A significant disadvantage for the star topology is that the generator 1 becomes a single point of failure for the complete network. If for any reason generator one trips, the control of the entire network of micro-grids will be lost and the consensus will fail. Making another generator the leader would require all the other generators to have a communication link with that new lead generator as well, which doubles the cost of the communication system. 


\subsection{Performance of Consensus Control with Communication Time Delays}

We earlier discussed that data transfer in reality is not continuous and takes time to travel from one place to another. Hence the effects of communication are also simulated and the performance is shown. In this chapter, loop topology has been used to demonstrate its effects.

\subsubsection{Case I- 0.5 Second Communication Delay}
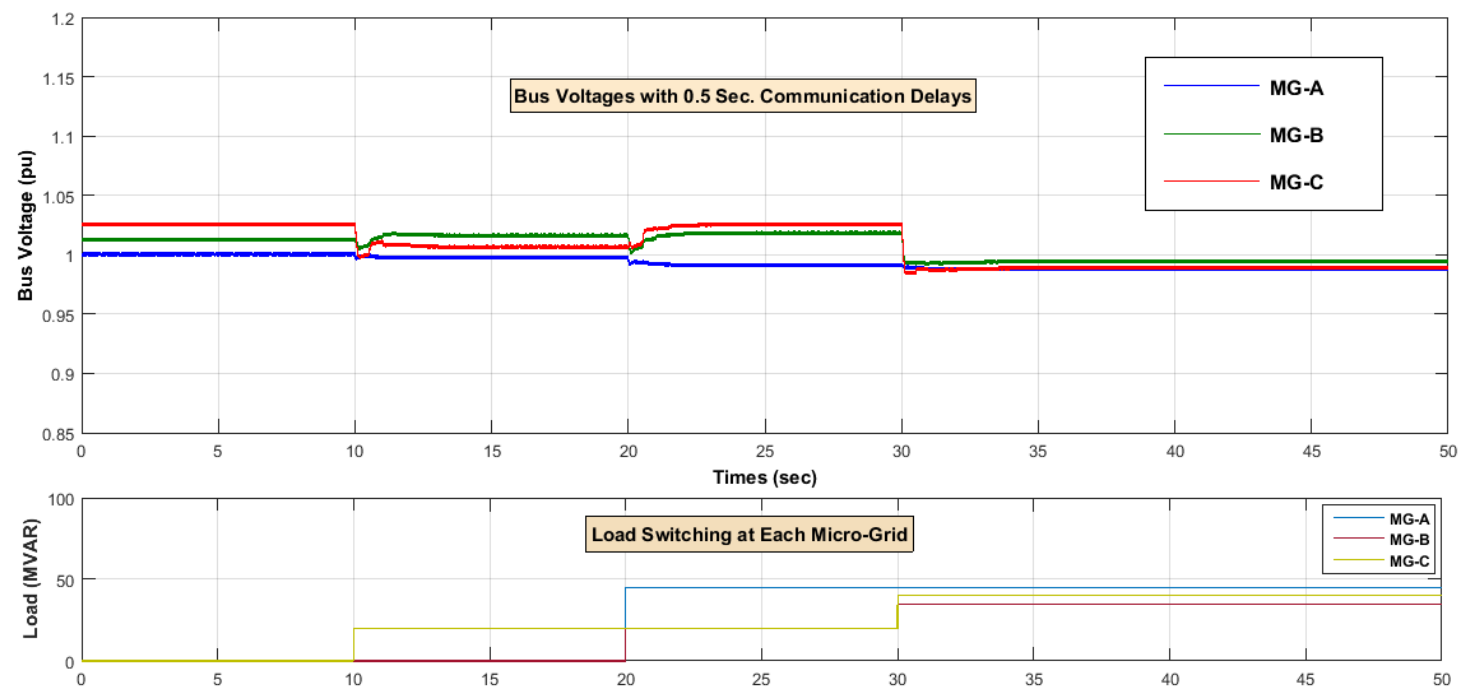

Figure 4.8: Consensus Algorithm with a communication delay of $0.5 \mathrm{sec}$

As shown in figure 4.8, a communication time delay of 0.5 seconds results in the consensus taking approximately 3.5 seconds to reach an agreement. The overall voltage profile remains the same. 


\subsubsection{Case II- 1 Second Communication Delay}
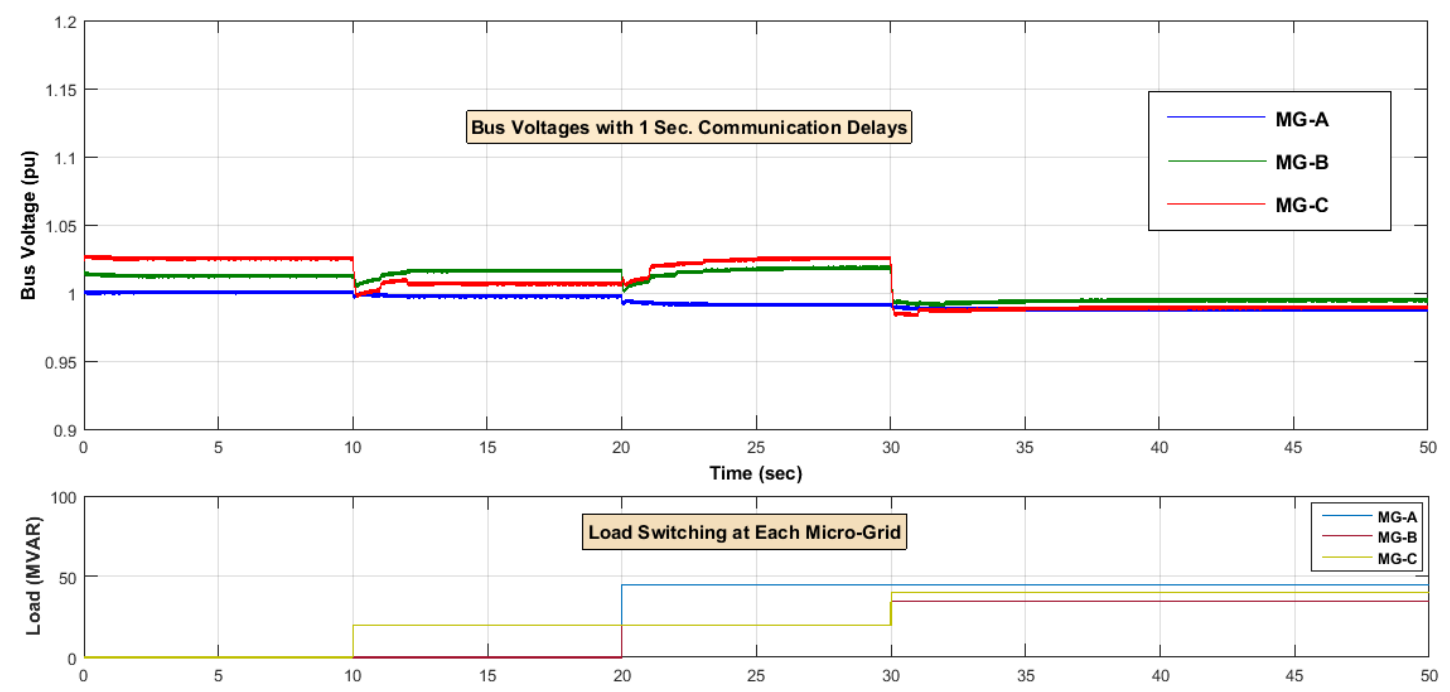

Figure 4.9: Consensus Algorithm with a communication delay of $1 \mathrm{sec}$

Figure 4.9 shows that a time delay of 1.0 second results in a consensus time of around 6.0 seconds and hence as the delay time doubles, the time that the consensus takes to reach an agreement also approximately doubles. The eventual voltage consensus remains the same and within the tolerance range but now the data reaching in time steps can easily be seen in the voltage profile of the bus voltages. 


\subsubsection{Case III- 3 Seconds Communication Delay}
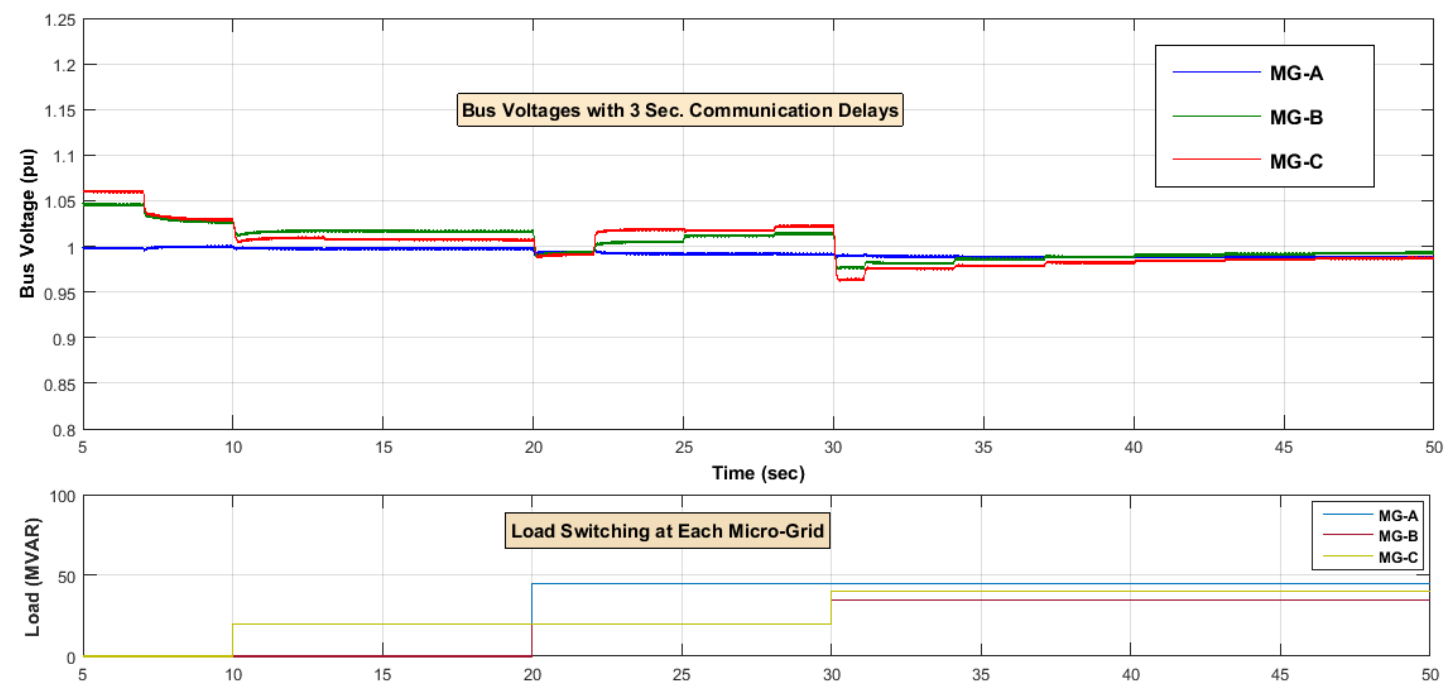

Figure 4.10: Consensus Algorithm with a communication delay of $3 \mathrm{sec}$

With a delay time of 3.0 seconds the time it takes to reach a consensus among the generators significantly increases to 9.1 seconds, as shown in figure 4.10. The voltage profile is also now in steps rather than the smooth transition seen earlier due to the data reaching after a large time delay of 3.0 seconds. The initial bus voltages can be ignored and is due to the initialization of the micro-grids having the same delay.

There is no guidelines or standards yet available to give guidelines on the allowable limits for the time the agents need to converge on a consensus time but the standards do restrict the bus voltages to be within $\pm 5 \%$ if following IEEE Standards 1547 or even $\pm 5 \%$ to $\pm 10 \%$ if following IEC standards 60038 [35-38]. Hence the decision comes down to the type of load application the micro-grid is providing power to since if the frequency of inductive load switching is high, then a faster 
consensus time would be required and vice versa. As a guide, we would recommend that the consensus time should be half the time of the typical mean time of load switching.

\subsection{Effect of Generator Tripping on the Performance of Consensus Control}

Generators can trip anytime due to a significant electrical disturbance or fault within the generators themselves or even on their respective buses and therefore it is important to simulate the probable scenario of generator tripping.

\subsubsection{Case I- Generator 3 Trip}

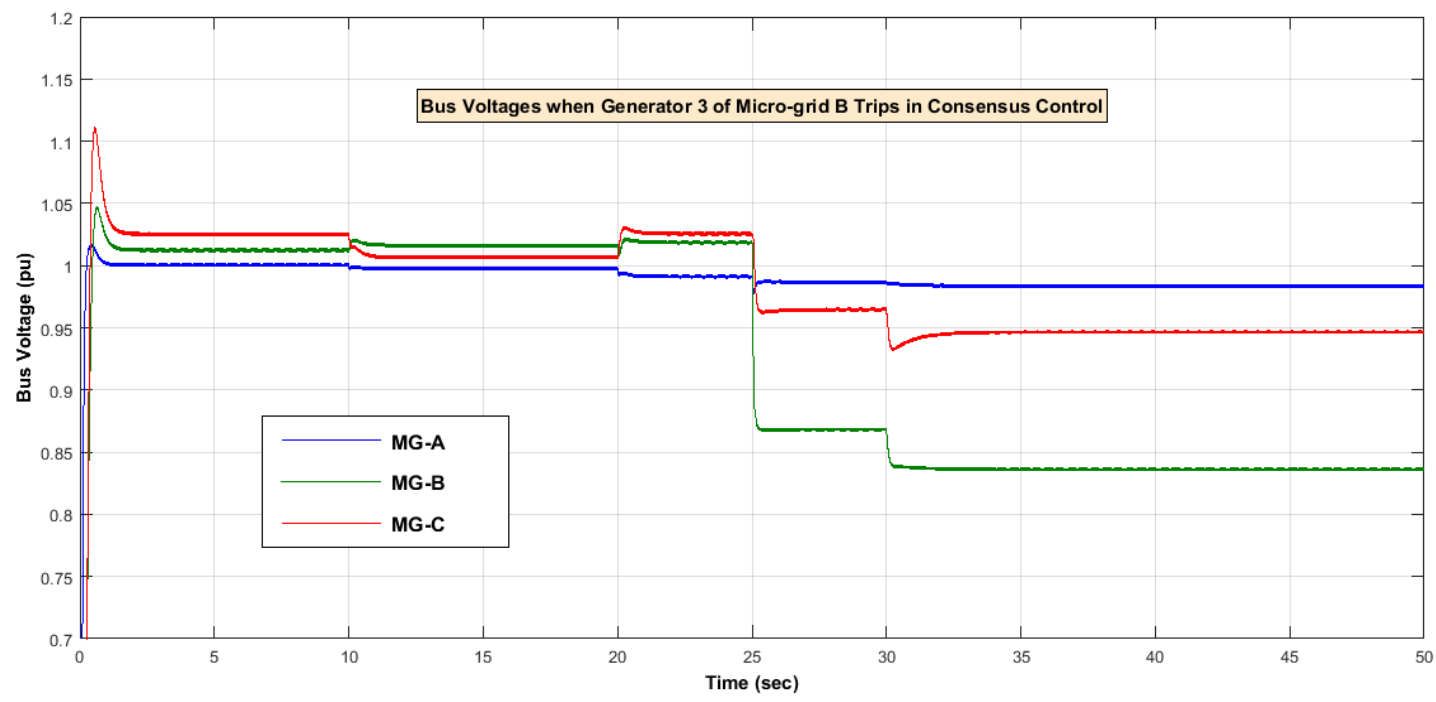

Figure 4.11: Effect of tripping of a generator in consensus control 
The simulation results in figure 4.11 shows us the effects of generator 3 of microgrid B tripping at 25 seconds of the simulation under loop topology as shown in figure 4.4. The voltages of micro-grid B and micro-grid C sharply decreases. This is because all three micro-grids are still connected with one another and with the tripping of one of the generators, the other generators are still coordinating in the consensus algorithm and therefore providing the same amount of reactive power set by the isochronous generator 1 . Since the set point of generator 1 does not vary much, generator 4 of micro-grid $B$ is not able to take on the reactive load left by the tripping, resulting in the sharp drop of bus voltage. Since micro-grid $C$ is directly connected to micro-grid $B$, it tries to provide the reactive power deficit but is not successful due to the same reasons and hence the bus voltage of micro-grid $C$ also significantly sags.

\subsubsection{Case II- Generator 3 Trip: Corrective Control}

It is obvious that the consensus algorithm does not perform if one of the generators trip and hence a corrective control needs to be put in place to ensure that the bus voltages of the micro-grids are not disturbed, otherwise the two micro-grids without the isochronous generators will trip as well. Therefore, a proposed approach is to have an interlocking of each generator with the bus-ties connecting the respective micro-grid of the generator. In case a generator trips, the bus-ties associated with its micro-grid open as well, shifting the micro-grids from the consensus control to the islanding mode in which each micro-grid has one isochronous generator to stabilize the bus voltage of the micro-grid. The simulation in figure 4.12 is to show if the interlocking would work or not. Generator 3 is again tripped at 25 seconds of the simulation. 


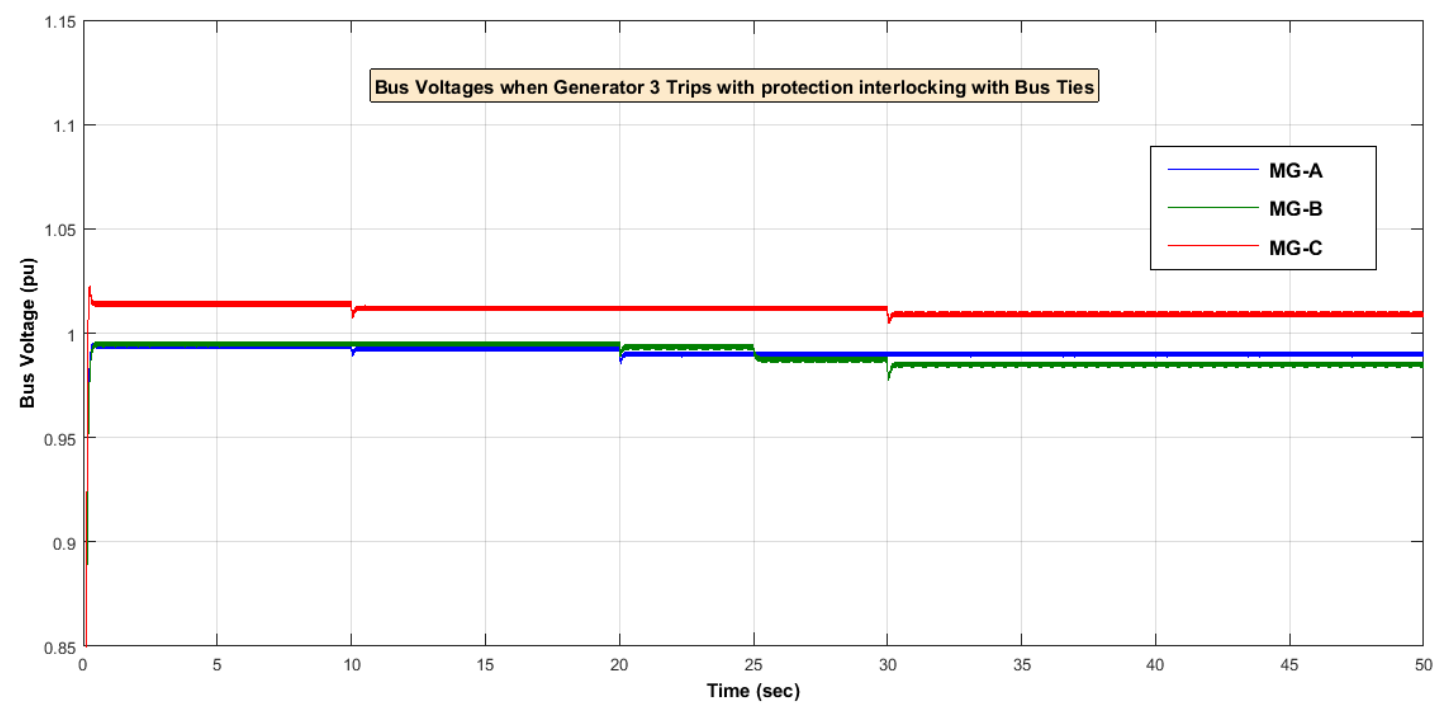

Figure 4.12: Corrective control due to the tripping of a generator

As figure 4.12 shows, the shift-over of control is smooth with no transient fluctuations or significant voltage drop. Micro-grid B is slightly affected by the trip but instantly maintains its bus voltage again with no effect on the other two microgrids. Hence this protection control method proves to be quite efficient and increase the reliability of the overall system. The bus-ties can be closed again when all the generators return to service.

\subsection{Sensitivity Analysis}

It is necessary to check the sensitivities of the system and the effects that it might have on the consensus control. The characteristic values that may have any impact on the consensus are the droop values of the generators, the time step used in the consensus algorithm and the load variations of the micro-grids. If the discrete 
consensus algorithm is checked again, the time step multiplies with the initial droop value, resulting in a constant value that can be called the 'consensus droop'. Since the change in the original droop or the time step will result in the same effect, we have lumped the two together and shown simulations of the varying consensus droop. The source impedances and the impedances of the interconnecting lines are also not being separately considered since they have minimal effect on the power transfer in our model and behave just like an inductive load. Therefore, their effects have been considered together with the inductive load variations at each micro-grid. Again, the loop topology of figure 4.4 , has been used to simulate the effects.

\subsubsection{Case I- Consensus Droop Decrease}
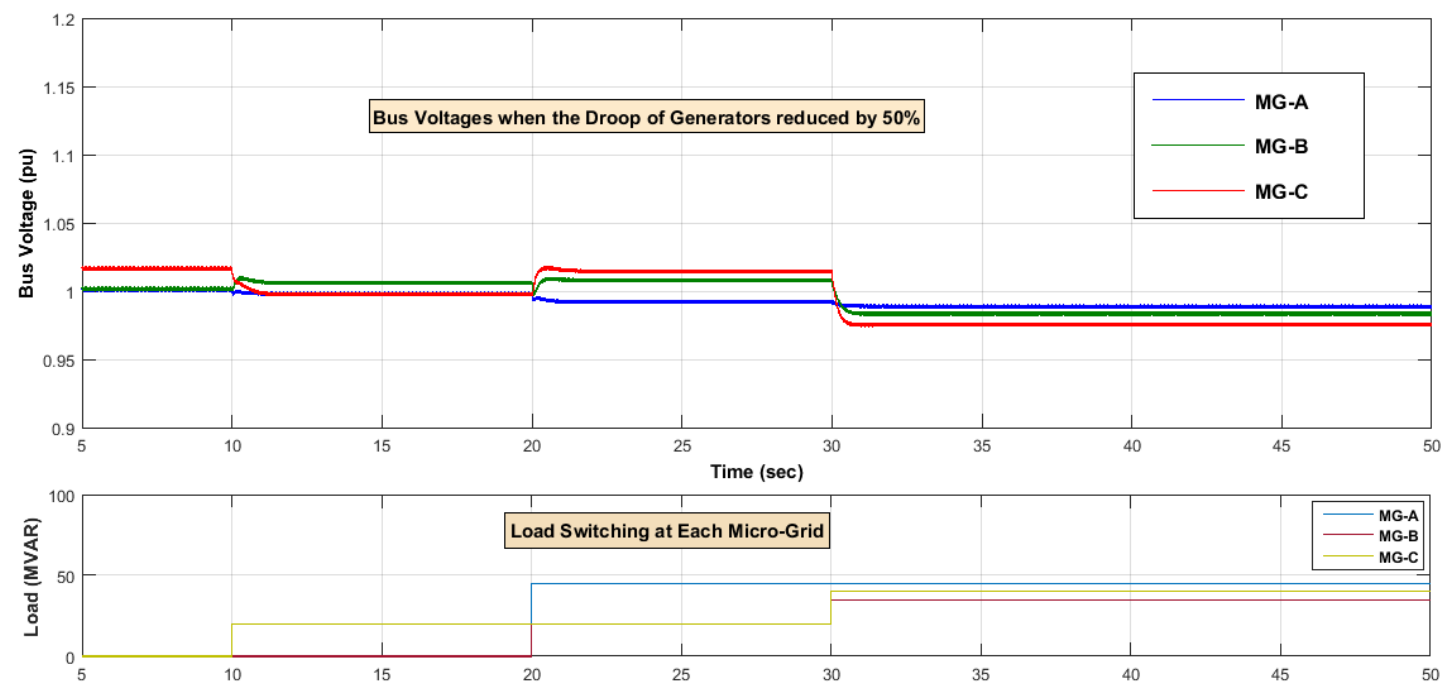

Figure 4.13: Effects of consensus droop decrease by $50 \%$

Figure 4.13 shows that when the consensus droop value is decreased by $50 \%$ the convergence time slightly increases to 2.0 seconds. The consensus convergence values as well as the voltage profile of each micro-grid bus remains the same. 


\subsubsection{Case II- Consensus Droop Increase}
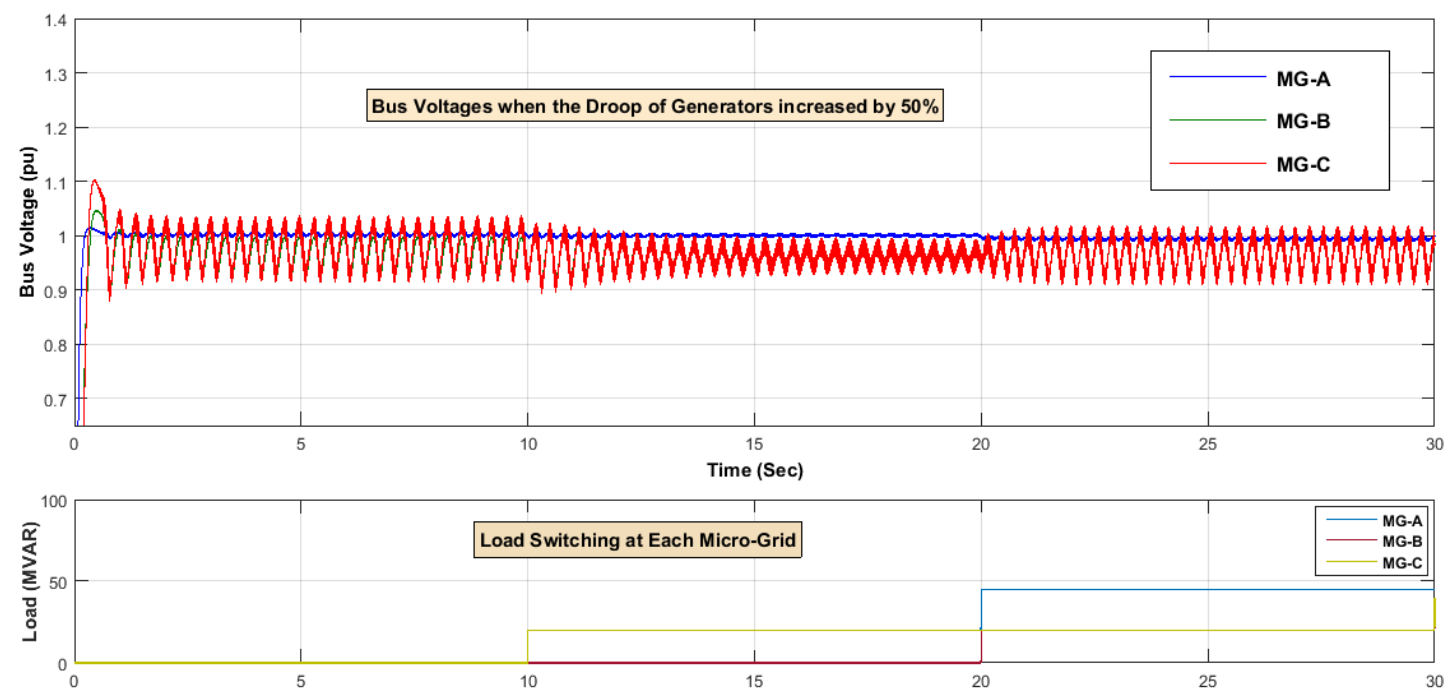

Figure 4.14: Effects of consensus droop increase by $50 \%$

The simulation results in figure 4.14 shows that, if the value of consensus droop is increased by $50 \%$, the performance of the controller degrades and the consensus is not able to converge. Instead severe voltage fluctuations can be observed with a damping effect in the middle due to load addition on micro-grid $A$ but again worsens after the switching of the second set of loads at 20 seconds.

Hence it can be deduced that the droop values used for modelling this algorithm are already near the higher end of the allowable limit and further increase might degrade the consensus algorithm. Furthermore, proper droop setting is necessary for the convergence of the consensus system. 


\subsubsection{Inductive Load Addition}

Previous impedance load additions were done in such a way that two loads were added to different combinations of two micro-grids simultaneously. To check the actual sensitivity of load to each micro-grid, inductive load of 20 MVAR per phase will be switched in at each bus separately. Micro-grid A will have the load switched at 10 seconds, micro-grid $B$ at 20 seconds and micro-grid $C$ at 30 seconds.

Addition of inductive load at micro-grid $A$, decreases the bus voltage of micro grid $A$ by just 0.003 pu but results in an increase of voltage at micro-grid $B$ by 0.017 pu and micro-grid C by $0.024 \mathrm{pu}$. This is because even though the inductive load has been added to Micro-grid A, its bus voltage remains somewhat steady due to the presence of the isochronous generator but its reactive power increases.
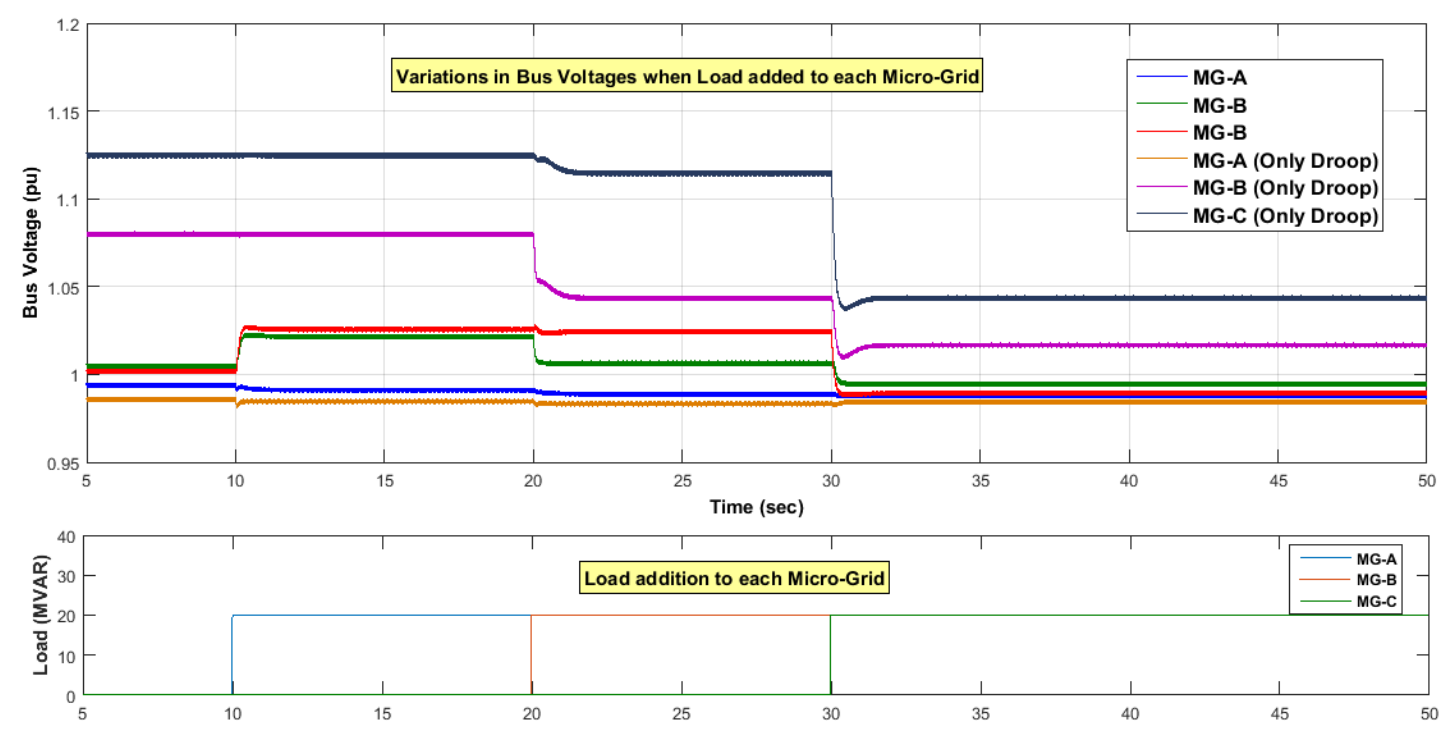

Figure 4.15: Voltage drop on each bus when 20 MVAR is added to each Micro-grid

This increase in reactive power is also translated to the other generators on microgrid $B$ and $C$ but the effect of the voltage drop due to the addition of load is not seen by them. Hence the further the micro-grid is from the load addition; the more 
voltage increase it witnesses. Still all the voltages remain within the allowable limits of bus voltages. This is not the case when the micro-grids are not working in consensus. The voltage of micro-grid A decreases slightly but the voltages of microgrid $B$ and $C$ are at unacceptable values of 1.08 pu and 1.126 pu.

The next addition inductive load is on micro-grid $B$ which results in a voltage drop of 0.015 pu on the very same bus. The voltage drop on micro-grid $A$ is again small, that is $0.002 \mathrm{pu}$ and on micro-grid C the voltage drop is also of just $0.001 \mathrm{pu}$. This load addition has minimal effect on the other micro-grids due to its position in the middle and hence the two micro-grids share the burden. In the case of no consensus, the voltage drop on micro-grid B is $0.037 \mathrm{pu}$ and the voltage drop on micro-grid $\mathrm{C}$ is of $0.01 \mathrm{pu}$.

The last addition of inductive load at micro-grid C causes a voltage drop of $0.036 \mathrm{pu}$, the largest drop, mainly because the micro-grid is the furthest from the isochronous generator. Micro-grid B also witnesses a significant drop of $0.012 \mathrm{pu}$ due to the same reasons but micro-grid A only has a voltage drop of just $0.001 \mathrm{pu}$. In the case of simple droop, the voltage drop on micro-grid B is $0.027 \mathrm{pu}$ and the voltage drop on micro-grid C is $0.071 \mathrm{pu}$. Voltages of each micro-grid in consensus control remain within the allowable range of voltage throughout the simulations but the voltages under simple droop go beyond the tolerance range.

\subsection{Summary}

The simulations in this chapter prove that the proposed consensus algorithm control works efficiently in not only ensuring that the reactive power is equally shared among the distributed generators of the micro-grid clusters but also that the voltage remains stabilized within in the acceptable range throughout the micro-grid cluster 
buses. It can be seen that the communication topology and the communication delays are a significant aspect in the performance of the consensus algorithm. The loop topology proves to be the most effective as it provides the highest level of connectivity between the generators. The tripping of a generator can deteriorate the effectiveness of the consensus algorithm but this can easily be avoided if proper interlocking protection strategy is employed. Finally, the sensitivities of the system are discussed in which the load variations and the particular value of consensus droop setting is brought up in focus. The variations due to these sensitivities are much less due to the implementation of consensus control yet still are significant and must be taken into account during the planning and designing phase of a microgrid cluster studies with consensus control. 


\section{Chapter 5 Conclusion and Future Work}

In this thesis, the use of consensus algorithm for an effective sharing of reactive power between distributed generators has been proposed and simulated. The need for such kind of reactive power sharing techniques is of utmost importance for a reliable and stable working of a micro-grid cluster since the demand and penetration of distribution generators in our present power system is increasing day by day. The conventional droop control system, even though seems simpler, but fails to maintain an equal, autonomous power sharing between the generators.

\subsection{Chapter Summary}

- Chapter 1 discussed the importance and motivation behind this thesis research and the problems faced in the control micro-grid clusters. The history of consensus algorithm was also discussed as well as the studies conducted in power system in which consensus algorithm have been used. A brief comparison of different types of micro-grid control methods has also been done. Finally, the objective and goals were listed in that chapter.

- Chapter 2 covered the essential background theory of the micro-grid system involved and the electrical equations associated with them. It also introduced and explained the concept of graph theory and how the consensus algorithm functions.

- Chapter 3 explained the modelling of the micro-grid clusters as well as the detailed functionality of the proposed consensus control system. This 
chapter also mentioned the typical issues faced when modelling a micro-grid using commercial power flow software and the assumptions taken to model the micro-grid cluster similar to the real case scenario.

- Chapter 4 presented the simulation case studies under various communication topologies and communication delays. The case studies also included the base case, where there was no communication between the distributed generators and also the fault case in which one generator trips. Finally, the sensitivity analysis was done and the overall performance of the consensus control algorithm was discussed.

\subsection{Contributions of Thesis}

The main contributions of this thesis can be highlighted as following:

1. It has been clearly shown that the consensus control algorithm efficiently works, can be easily implemented and is cost effective. All the generators involved, easily converge to the desired value of reactive power within 1.5 seconds of the load disturbance and keep all the micro-grid bus voltages within the tolerance range of the voltages.

2. This thesis has also verified that the topology used for the communication between distributed generators play a vital role in the performance of the consensus control. To have the fastest response, it was necessary to have the highest connectivity level between the generators. This was evident in the differences of convergence time in the three common communication topologies considered in this thesis. Out of them, the looped topology gave us the fastest response and hence was the most efficient of all the communication networks. 
3. The significance of a suitable and reliable communication channel system was also demonstrated by simulating the consensus control under different communication delays. It was easily proved that a faster communication system would converge faster and hence the need for an optimal communication channel. Since a better communication channel would result in faster data transfer between the distributed generators and thus resulting in an overall faster convergence of all the generators.

4. The fault case, in which any of the generators trips, has also been studied. Even though the performance of the consensus algorithm degraded when one generator tripped, it was proved that the system can remain stable if it incorporates simple control strategies of opening the micro-grid bus-ties using interlocks. Even though this takes out the generators from the consensus control and forces the micro-grids into islanding mode but this keeps the overall system stable and free from any voltage fluctuations, which is the main concern of the end consumer.

5. This thesis has also done the sensitivity analysis of the micro-grid cluster under consensus control. It was shown that the variations in generator consensus droop has an effect on the convergence rate of the consensus algorithm and thus careful tuning of generator droop parameters is of paramount importance for the optimal convergence rate. If the droop value used is too large, then generators would not converge and will start 'hunting' around the mean value of reactive power. If the droop value is too small, then the convergence rate would decrease and will result in a longer time for the generator to reach consensus. Apart from droop, large load variation analysis was also done to see the voltage change of the micro-grid bus accordingly. Therefore, even though the consensus algorithm worked perfectly, large load changes still affected the bus voltage and beyond the threshold value, completely destabilized the microgrid voltage altogether. 


\subsection{Future Work}

This study has been conducted without the aspects of active power flow dynamics and even though it is sufficient for the purpose of micro-grid voltage stability, the overall dynamics of the complete electrical system is yet to be studied. In future, if the active power and frequency dynamics are included, then a more complex yet realistic analysis can be done including the effects of inertial loads and frequency variations. The respective study can be further verified by increasing the model to incorporate a larger number of micro-grids and confirm if the convergence time remains the same. This study can further be increased and be made even more realistic by including the modelling of power electronic invertors for the distributed generators and include the issues involving them, including control issues, harmonics, reliability and further communication complexities.

As a micro-grid can have distributed generators of different size and ratings, another case that can be studied is when one of the distributed generators gives out maximum power and saturates. In this case that distributed generator should fix at its maximum output dispatch and the other distributed generators should work on dividing the remaining power among themselves through consensus control.

Proper fault analysis should also be done in this control scheme, since as the generators are working based on consensus feedback, they can theoretically increase the electrical fault levels. Therefore, the protection system should also be

properly studied, coordinated and designed under this control algorithm, so that electrical system can sustain and isolate any fault without significant damages.

Since the consensus droop value has a significant impact on the performance of the consensus algorithm, the consensus droop can be worked upon to make it adaptive and change its value depending upon the prevailing conditions of the micro-grid. 


\section{References}

[1] E. Martinot, "Grid Integration of Renewable Energy: Flexibility, Innovation, and Experience," Annual Review of Environment and Resources, vol. 41, pp. 223-251, 2016.

[2] R. Karki and R. Billinton, "Reliability/cost implications of PV and wind energy utilization in small isolated power systems," IEEE Transactions on Energy Conversion, vol. 16, pp. 368-373, 2001.

[3] S. Corneli, S. Kihm, and L. Schwartz, "Electric Industry Structure and Regulatory Responses in a High Distributed Energy Resources Future," 2015.

[4] L. Y. Lu and C. C. Chu, "Consensus-Based Droop Control Synthesis for Multiple DICs in Isolated Micro-Grids," IEEE Transactions on Power Systems, vol. 30, pp. 22432256, 2015.

[5] L. Y. Lu and C. C. Chu, "Consensus-Based Secondary Frequency and Voltage Droop Control of Virtual Synchronous Generators for Isolated AC Micro-Grids," IEEE Journal on Emerging and Selected Topics in Circuits and Systems, vol. 5, pp. 443-455, 2015.

[6] Y. Fan, G. Hu, and M. Egerstedt, "Distributed Reactive Power Sharing Control for Microgrids With Event-Triggered Communication," IEEE Transactions on Control Systems Technology, vol. 25, pp. 118-128, 2017.

[7] R. Olfati-Saber, J. A. Fax, and R. M. Murray, "Consensus and Cooperation in Networked Multi-Agent Systems," Proceedings of the IEEE, vol. 95, pp. 215-233, 2007.

[8] N. Rahbari-Asr, Y. Zhang, and M.-Y. Chow, "Consensus-based distributed scheduling for cooperative operation of distributed energy resources and storage devices in smart grids," IET Generation, Transmission \& Distribution, vol. 10, pp. 1268-1277, 2016.

[9] H.-L. Choi, L. Brunet, and J. P. How, "Consensus-based decentralized auctions for robust task allocation," IEEE transactions on robotics, vol. 25, pp. 912-926, 2009.

[10] W. Ren and R. W. Beard, Distributed consensus in multi-vehicle cooperative control: Springer.

[11] B. Peng, Z. Zhao, G. Han, and J. Shen, "Consensus-based sparse signal reconstruction algorithm for wireless sensor networks," International Journal of Distributed Sensor Networks, vol. 12, p. 1550147716666290, 2016/09/01 2016.

[12] B. A. S. J and M. Jamshidi, "Consensus-based and network control of UAVs," in 2010 5th International Conference on System of Systems Engineering, 2010, pp. 1-6.

[13] R. R. Kolluri, I. Mareels, T. Alpcan, M. Brazil, J. d. Hoog, and D. Thomas, "Power sharing correction in angle droop controlled inverter interfaced microgrids," in 2015 IEEE Power \& Energy Society General Meeting, 2015, pp. 1-5.

[14] L. Chen, Y. Wang, L. Yang, Y. Si, T. Chen, and S. Mei, "Consensus control strategy with state predictor for virtual synchronous generators in isolated microgrid," in 2016 IEEE International Conference on Power System Technology (POWERCON), 2016, pp. 1-5. 
[15] Y. Xu, W. Liu, and J. Gong, "Stable multi-agent-based load shedding algorithm for power systems," IEEE Transactions on Power Systems, vol. 26, pp. 2006-2014, 2011.

[16] Z. Zhang and M.-Y. Chow, "Convergence analysis of the incremental cost consensus algorithm under different communication network topologies in a smart grid," IEEE Transactions on Power Systems, vol. 27, pp. 1761-1768, 2012.

[17] J. W. Simpson-Porco, F. Dörfler, F. Bullo, Q. Shafiee, and J. M. Guerrero, "Stability, power sharing, \&amp; distributed secondary control in droop-controlled microgrids," in 2013 IEEE International Conference on Smart Grid Communications (SmartGridComm), 2013, pp. 672-677.

[18] P. Kundur, N. J. Balu, and M. G. Lauby, Power system stability and control vol. 7: McGraw-hill New York, 1994.

[19] Y. Xiu, Z. Xiang, Y. Fei, and Z. Hai-yang, "A research on droop control strategy and simulation for the micro-grid," in 2011 International Conference on Electrical and Control Engineering, 2011, pp. 5695-5700.

[20] J. Liu, Y. Miura, and T. Ise, "Comparison of Dynamic Characteristics Between Virtual Synchronous Generator and Droop Control in Inverter-Based Distributed Generators," IEEE Transactions on Power Electronics, vol. 31, pp. 3600-3611, 2016.

[21] Q.-C. Zhong, "Robust droop controller for accurate proportional load sharing among inverters operated in parallel," IEEE Transactions on Industrial Electronics, vol. 60, pp. 1281-1290, 2013.

[22] Q. Sun, R. Han, H. Zhang, J. Zhou, and J. M. Guerrero, "A multiagent-based consensus algorithm for distributed coordinated control of distributed generators in the energy internet," IEEE transactions on smart grid, vol. 6, pp. 3006-3019, 2015.

[23] J. He and Y. W. Li, "An Enhanced Microgrid Load Demand Sharing Strategy," IEEE Transactions on Power Electronics, vol. 27, pp. 3984-3995, 2012.

[24] M. Yazdanian and A. Mehrizi-Sani, "Distributed Control Techniques in Microgrids," IEEE Transactions on Smart Grid, vol. 5, pp. 2901-2909, 2014.

[25] H. Mahmood, D. Michaelson, and J. Jiang, "Accurate Reactive Power Sharing in an Islanded Microgrid Using Adaptive Virtual Impedances," IEEE Transactions on Power Electronics, vol. 30, pp. 1605-1617, 2015.

[26] M. Falahi, K. Butler-Purry, and M. Ehsani, "Dynamic reactive power control of islanded microgrids," IEEE Transactions on Power Systems, vol. 28, pp. 3649-3657, 2013.

[27] C. Niannian and J. Mitra, "A decentralized control architecture for a microgrid with power electronic interfaces," in North American Power Symposium 2010, 2010, pp. 1-8.

[28] I. S. Bae and J. O. Kim, "Reliability Evaluation of Customers in a Microgrid," IEEE Transactions on Power Systems, vol. 23, pp. 1416-1422, 2008.

[29] R. H. Lasseter, "MicroGrids," in 2002 IEEE Power Engineering Society Winter Meeting. Conference Proceedings (Cat. No.02CH37309), 2002, pp. 305-308 vol.1.

[30] R. H. Lasseter, A. A. Akhil, C. Marnay, J. Stephens, J. E. Dagle, R. T. Guttromson, et al., "Integration of Distributed Energy Resources: The CERTS MicroGrid Concept," CERTS10/2003 2003.

[31] J. M. Guerrero, M. Chandorkar, T.-L. Lee, and P. C. Loh, "Advanced control architectures for intelligent microgrids-Part I: Decentralized and hierarchical control," IEEE Transactions on Industrial Electronics, vol. 60, pp. 1254-1262, 2013. 
[32] A. Bidram and A. Davoudi, "Hierarchical Structure of Microgrids Control System," IEEE Transactions on Smart Grid, vol. 3, pp. 1963-1976, 2012.

[33] S. Chapman, Electric machinery fundamentals: Tata McGraw-Hill Education, 2005.

[34] A. M. Bollman, "An experimental study of frequency droop control in a low-inertia microgrid," University of Illinois at Urbana-Champaign, 2009.

[35] I. E. Commission, "IEC 60038 Standard Voltages," IEC Central Office, Geneva, Switzerland, 2009.

[36] "IEEE Application Guide for IEEE Std 1547(TM), IEEE Standard for Interconnecting Distributed Resources with Electric Power Systems," IEEE Std 1547.2-2008, pp. 1217, 2009.

[37] "IEEE Guide for Design, Operation, and Integration of Distributed Resource Island Systems with Electric Power Systems," IEEE Std 1547.4-2011, pp. 1-54, 2011.

[38] "IEEE Guide for Monitoring, Information Exchange, and Control of Distributed Resources Interconnected with Electric Power Systems," IEEE Std 1547.3-2007, pp. 1-160, 2007. 


\section{Appendix A PSCAD Screenshots}

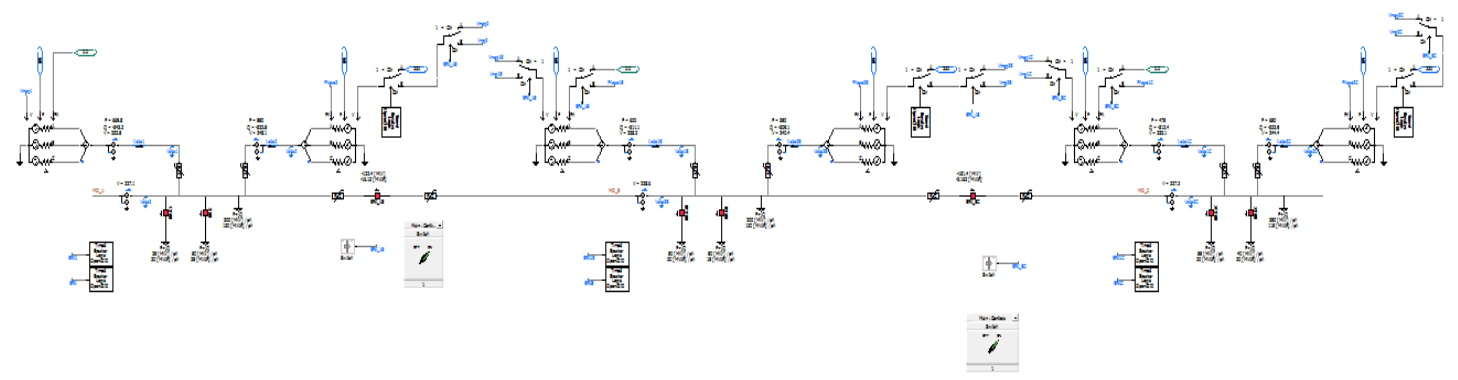

Figure A.1: Complete Micro-Grid Cluster Model in PSCAD

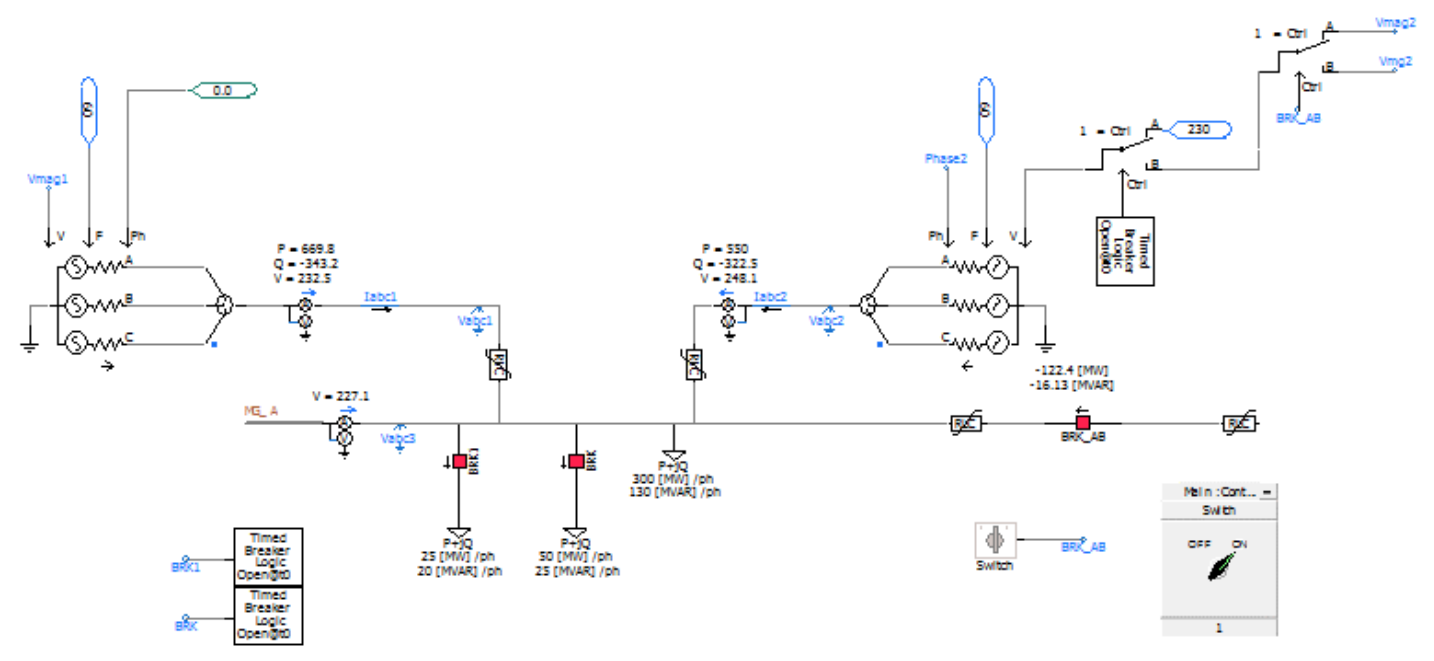

Figure A.2: Model of Micro-Grid A in PSCAD 


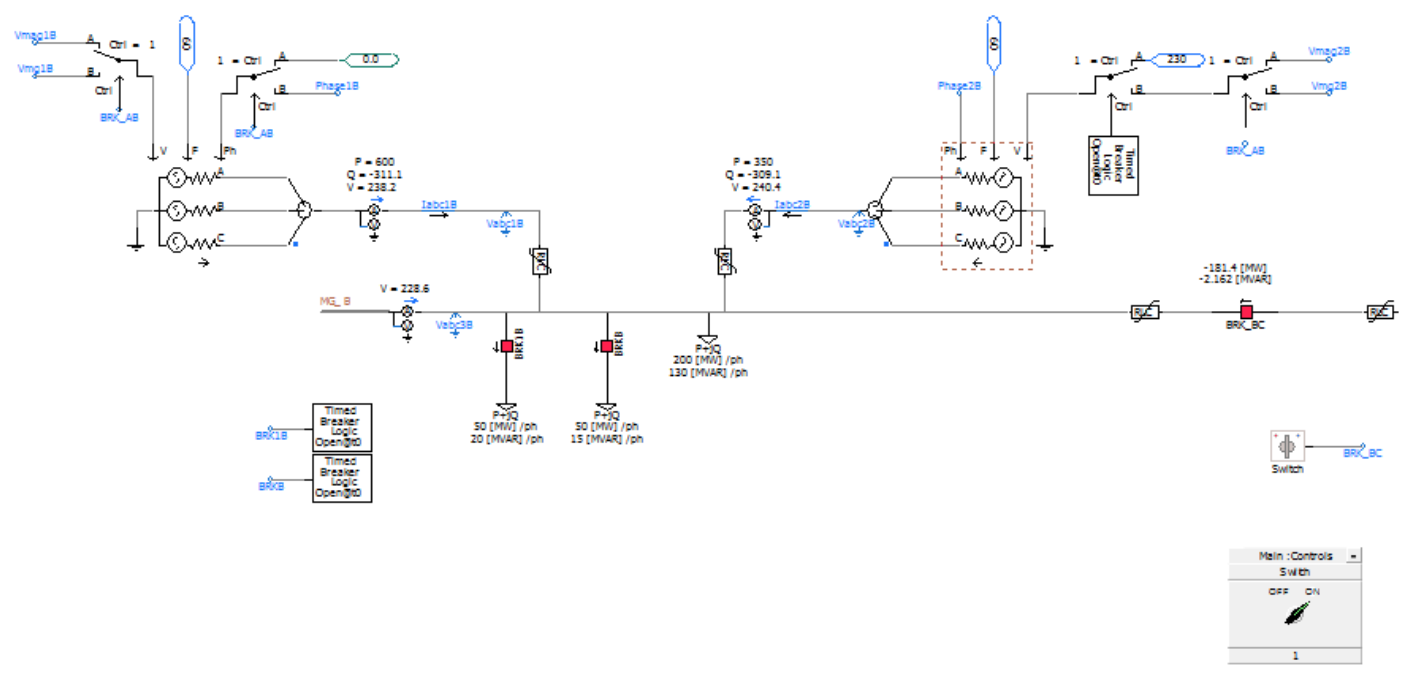

Figure A.3: Model of Micro-Grid B in PSCAD

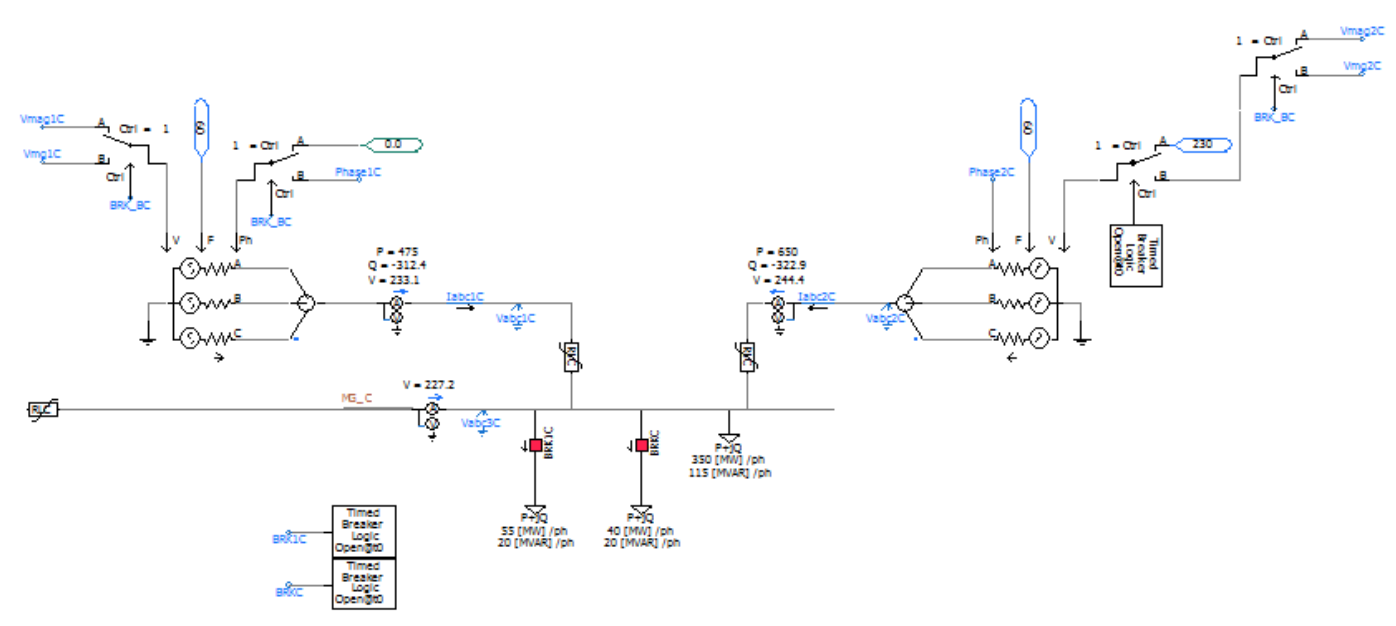

Figure A.4: Model of Micro-Grid C in PSCAD 


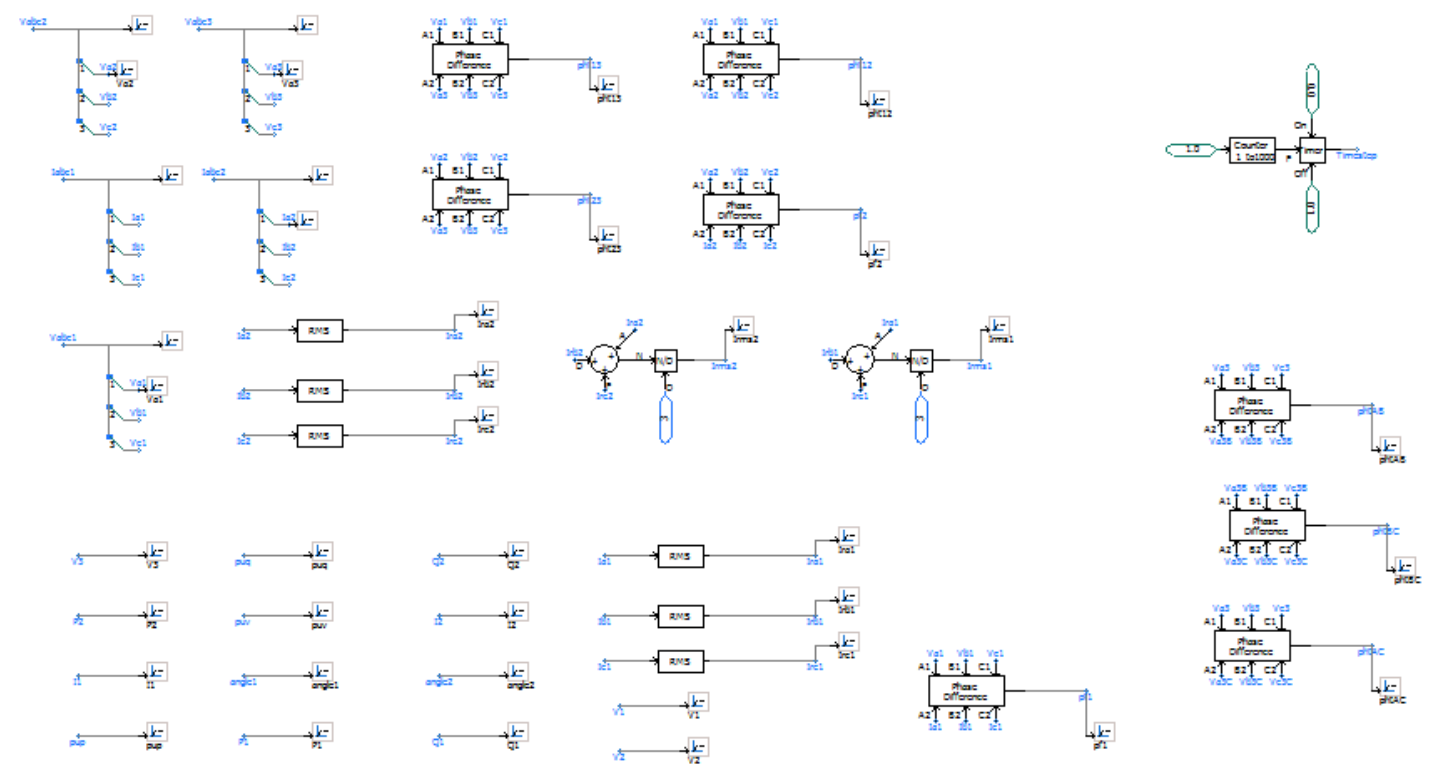

Figure A.5: Measurement blocks of Micro-Grid A

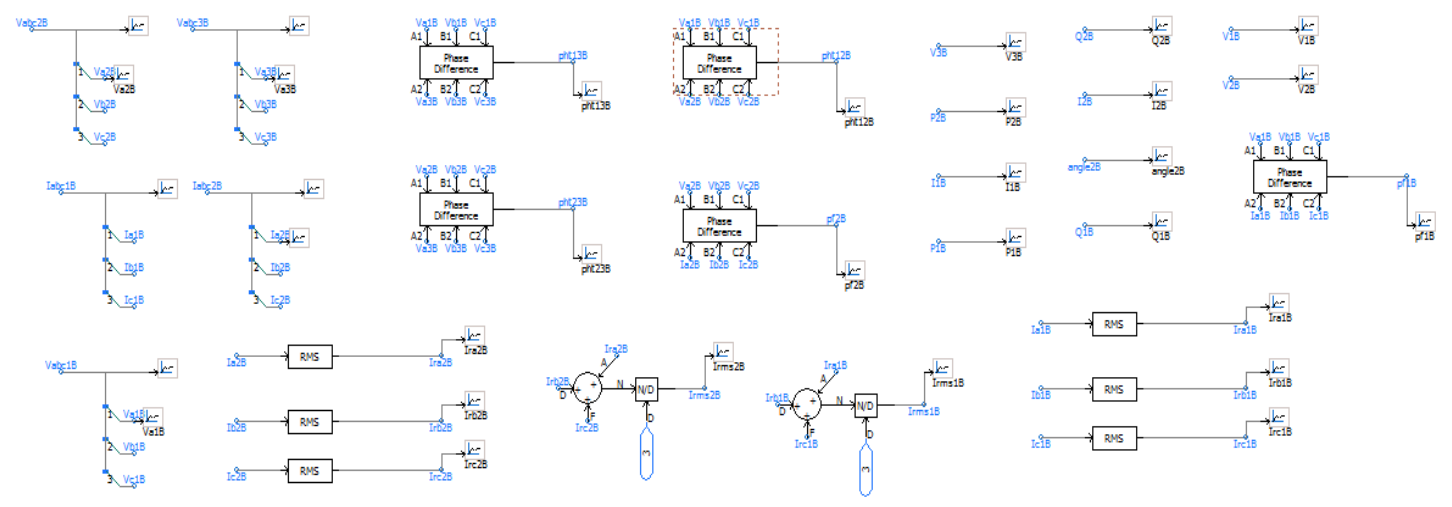

Figure A.6: Measurement blocks of Micro-Grid B 


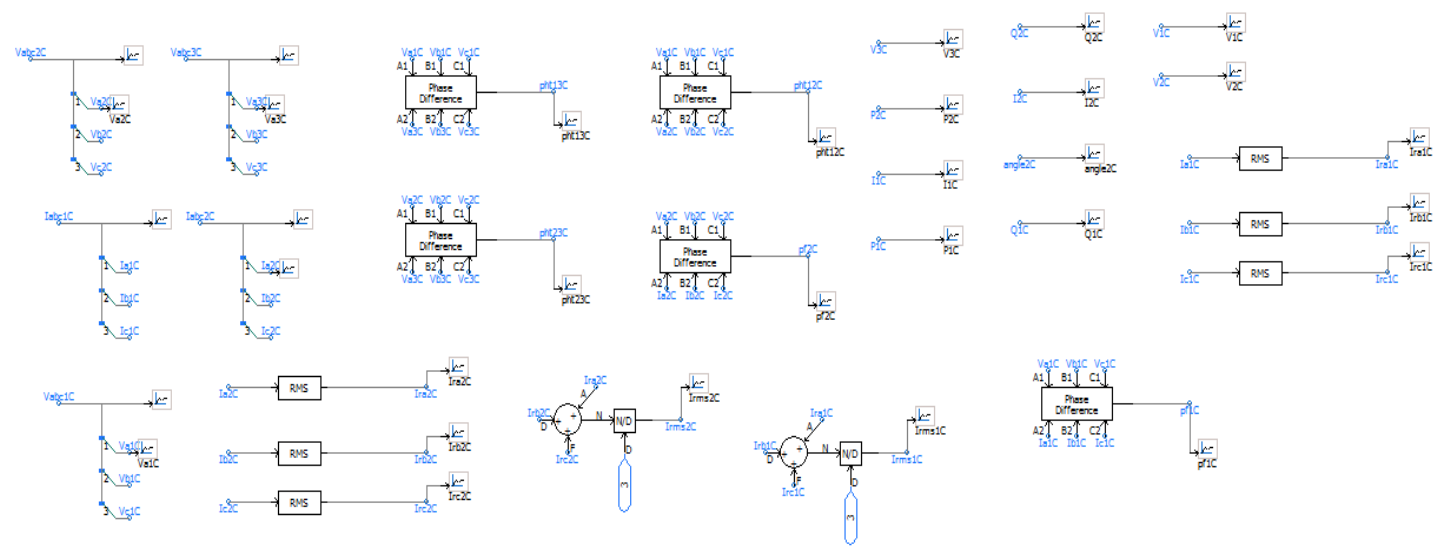

Figure A.7: Measurement blocks of Micro-Grid C
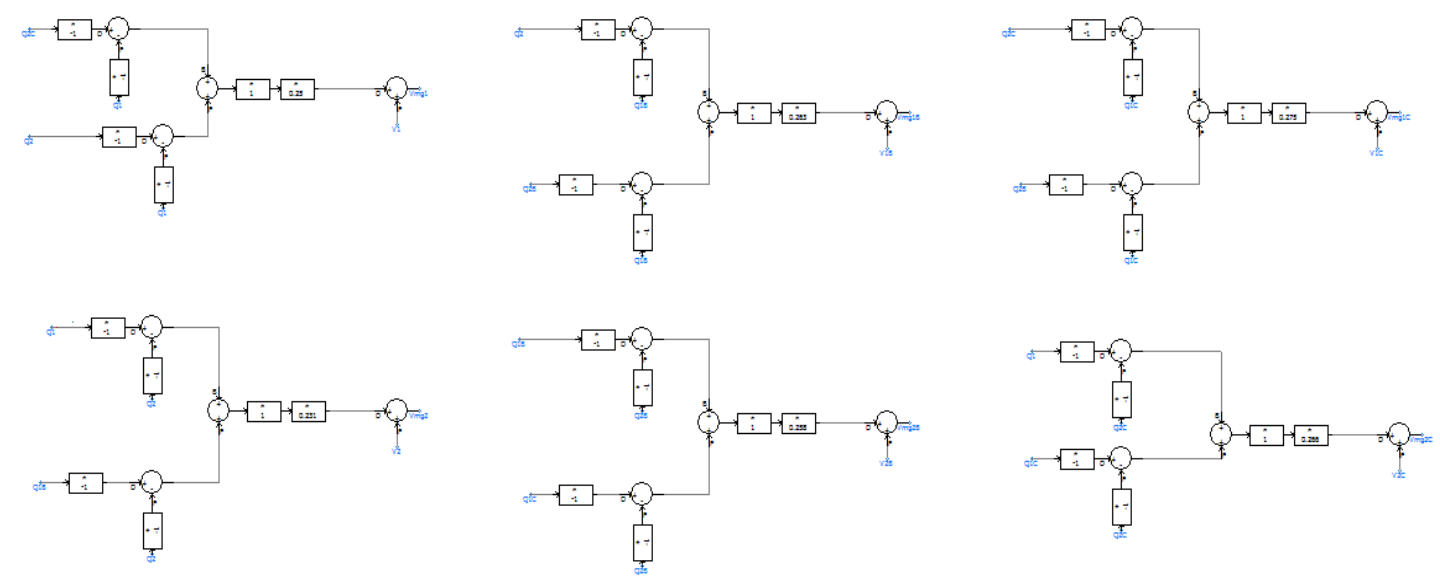

Figure A.8: Consensus reactive power control blocks 


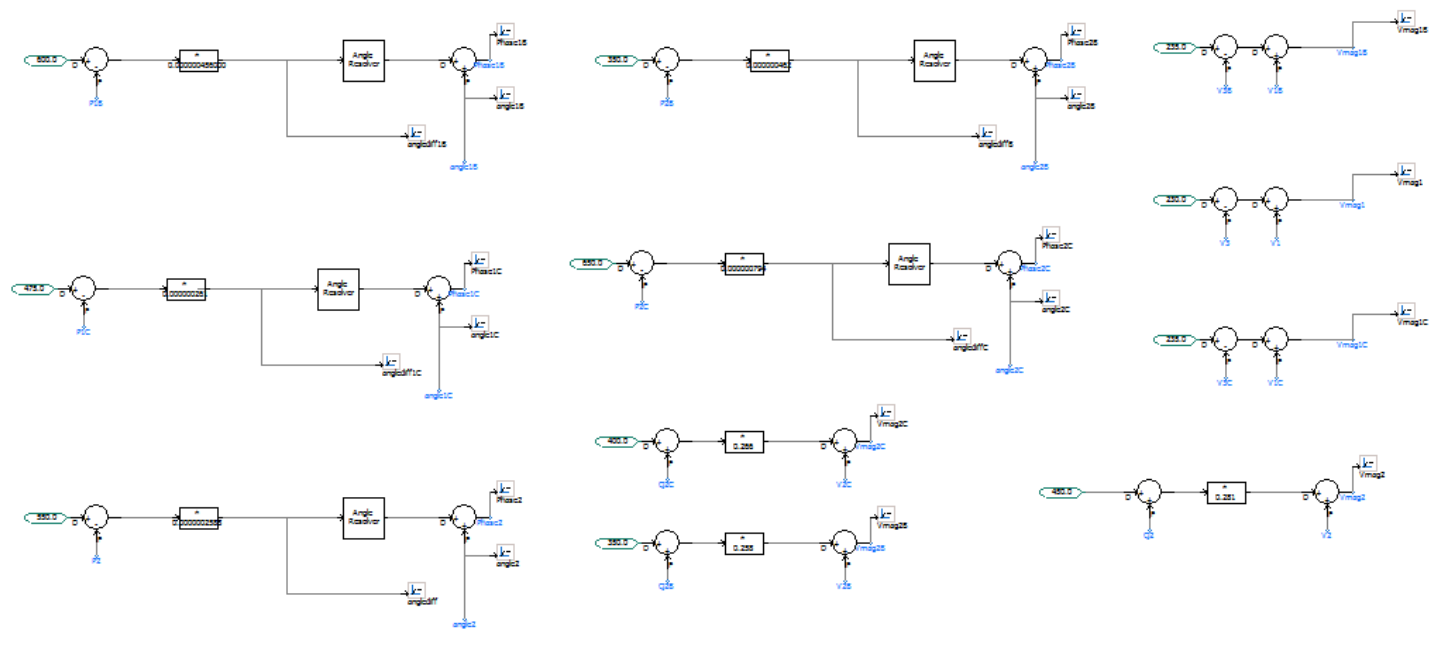

Figure A.9: Active power and Islanding mode control blocks 